\title{
ANÁLISIS DE LA GESTIÓN MONETARIA DEL BCE DESDE UN ENFOQUE ENDÓGENO
}

\section{ANALYSIS OF THE ECB'S MONETARY MANAGEMENT FROM AN ENDOGENOUS APPROACH}

\author{
LUIS CÁRDENAS DEL REY \\ Universidad Complutense de Madrid \\ luiscard@ucm.es \\ Fecha de recepción: octubre de 2013 \\ Fecha de aceptación: julio de 2014
}

\begin{abstract}
RESUMEN
El propósito de este trabajo es estudiar la política monetaria desarrollada por el Banco Central Europeo (BCE) en los últimos años, contrastándose la hipótesis de efectividad monetaria en el caso de la economía española. Para ello se sintetizan las principales medidas tomadas durante el período, especialmente, durante la evolución de la crisis de deuda, y se analiza el comportamiento de los principales indicadores. A diferencia de los trabajos realizados hasta la fecha, que consideran que la política monetaria no convencional ha cumplido sus objetivos, en la presente investigación se sostiene que ni ha beneficiado a la economía española ni ha tenido un efecto expansivo en la actividad económica, i.e., no ha afectado a los agregados monetarios sino al balance del banco central. La explicación del fenómeno se basa en un enfoque endógeno de la oferta monetaria y la necesidad de introducción de diferentes instrumentos de política monetaria y reforma socio-institucional para que la política monetaria sea efectiva.
\end{abstract}

Palabras clave: Banco Central Europeo, crisis financiera, medidas no convencionales, política monetaria.

\begin{abstract}
The aim of this paper is to study monetary policy developed by the European Central Bank (ECB) in recent years, contrasting the monetary effectiveness hypothesis in the case of the Spanish economy. This summarizes the main measures taken during the period, especially during the evolution of the debt crisis, and analyzes the behavior of the main indicators. In contrast to the papers carried out to date, which believe that non-standard monetary policy has met its objectives, in the present study argues that neither benefited nor the Spanish economy has had a expansionary effect on economic activity, i. e., didn't affect monetary aggregates but the central bank balance sheet. The explanation approach is based on an endogenous money supply and the need for introduction of different monetary policy instruments and socialinstitution reform that monetary policy is effective.
\end{abstract}

Key Words: European Central Bank, Financial crisis, Non-standard monetary policy, monetary policy. 
Cárdenas, Luís. Análisis de la gestión monetaria del BCE desde un enfoque de endógeno.

JEL: E10, E52, E58

\section{INTRODUCCIÓN}

La presente investigación tiene por objeto analizar la política monetaria desarrollada por el Banco Central Europeo (BCE) en los últimos años y sus consecuencias, mediante los efectos monetarios, para la economía española. Se enumeran las principales características del BCE y medidas tomadas a partir de la crisis financiera y la evolución de la crisis de deuda soberana. A diferencia de otros estudios se opta por un enfoque metodológico diferente basado en la teoría de la endogeneidad de la oferta monetaria, mediante el cuál se defiende que la política monetaria no convencional no ha tenido un efecto expansivo en la actividad económica puesto que no ha supuesto un aumento de los agregados monetarios sino que ha producido un incremento del balance del banco central. Por tanto, esta política no se refleja en la inflación y es consecuente con el principal objetivo del BCE, la estabilidad de precios a corto y largo plazo.

El trabajo se organiza de la siguiente forma: En primer lugar, en el presente punto se realiza una revisión de la literatura reciente sobre la actuación del Banco Central Europeo (BCE), y en concreto, sobre las políticas no convencionales, se argumenta que la visión dominante se encuentra en línea con el «Nuevo Consenso Macroeconómico», $\mathrm{NCM}^{1}$ (New Consensus View), siendo esta teoría inadecuada para instrumentar medidas de política económica, al considerar inexistentes los efectos monetarios y considerarlos potencialmente inflacionistas.

En segundo lugar, se detalla la estrategia monetaria seguida por el BCE, los instrumentos utilizados, su trayectoria de gestión pasada hasta las últimas medidas tomadas. Se distingue entre política monetaria convencional y no convencional, aunque desde un enfoque diferente al utilizado frecuentemente.

Tercero, se analizan los hechos estilizados observados en la economía monetaria, el conjunto de datos monetarios lo componen los principales agregados (M1, M2, M3) tanto en el caso de la Eurozona como en el de España; también el balance del Banco de España tiene un rol crucial para entender las medidas de política monetaria no convencionales. Posteriormente se analizan el comportamiento de los tipos de interés respecto al tipo de referencia oficial, la evolución de los precios desde el año 2007, y la correlación entre la masa monetaria respecto a la inflación y su incidencia en la producción industrial.

A continuación, siguiendo a Arestis (2012) se desarrollan los argumentos de un enfoque monetario endógeno y el papel de la política monetaria, así como la posibilidad de monetización de deuda. Finalmente, se resumen las aportaciones obtenidas y se realizan una serie de propuestas de política económica consecuentes con el desarrollo teórico y empírico posterior.

\footnotetext{
${ }^{1}$ En el presente trabajo se opta por distinguir entre dos corrientes de pensamiento que se definen explícitamente: el NCM y el enfoque endógeno. Evitando de esta forma tener que recurrir y explicar las diferentes etiquetas de clásico, neoclasicismo, monetarista, keynesiano, neokeynesiano, postkeynesiano, horizontalista, estructuralista, etc. que pueden dar lugar a confusión si no se acompañan de un desarrollo en profundidad de sus orígenes y desarrollos posteriores, lo cual no es objeto de este estudio.
} 
Cárdenas, Luís. Análisis de la gestión monetaria del BCE desde un enfoque de endógeno.

La motivación de este trabajo es que, sin duda, la política monetaria es uno de los aspectos más tratados en la macroeconomía, y dado el marco macroeconómico de estancamiento y recesión con posterioridad a la crisis financiera del 2007, se ha desarrollado una prolífica literatura sobre la política monetaria en tiempos de crisis, que incluso replantea las nociones básicas sobre las que se fundamentaba la política monetaria en las dos últimas décadas. Mishkin, (2011, p.2) señala nueve principios básicos que eran aceptados en todos los bancos centrales, estos son:

1) inflation is always and everywhere a monetary phenomenon; 2) price stability has important benefits; 3) there is no long-run tradeoff between unemployment and inflation; 4) expectations play a crucial role in the determination of inflation and in the transmission of monetary policy to the macroeconomy; 5) real interest rates need to rise with higher inflation, i.e., the Taylor Principle; 6) monetary policy is subject to the time inconsistency problem; 7) central bank independence helps improve the efficiency of monetary policy; 8) commitment to a strong nominal anchor is central to producing good monetary policy outcomes; and 9) financial frictions play an important role in business cycles.

Frente a este estado de fundamentos teóricos sobre los que desarrollar la política monetaria, Mishkin señala que se han de incorporar nuevos aspectos, estos nuevos puntos se centran en el sector financiero como agente fundamental para el desarrollo de la estrategia monetaria. Estas incorporaciones se sintetizan en: 1) la evolución del sector financiero tiene mayor impacto sobre el desarrollo económico de lo que se pensaba; 2) la macroeconomía tiene un comportamiento fundamentalmente no lineal; 3) la proximidad de los tipos de interés a cero es un problema mayor de lo que se estimaba (trampa de liquidez); 4) los costes derivados de quiebras financieras son excesivamente elevados; 5) la estabilidad de precios y producción no garantiza la estabilidad financiera.

No se observan grandes diferencias con el pensamiento clásico, como el laureado economista, y pilar de la Nueva Macroeconomía Clásica, Robert Lucas sostenía en su discurso de recogida del Premio Nobel, la alta correlación entre inflación y tasa de crecimiento de la masa monetaria demostraba con evidencia empírica la teoría cuantitativa del dinero defendida por los clásicos (Lucas 1996, p. 232):

the prediction that prices respond proportionally to changes in money in the long run, deduced by Hume in 1752 (and by many other theorists, by many different routes, since), has received ample - I would say, decisive - confirmation, in data from many times and places.

En esta línea y ante la especial gravedad de la evolución económica en los países de la periferia de la Eurozona, la gestión monetaria del Banco Central Europeo es objeto de un constante estudio, e incluso, se le señala entre el conjunto de circunstancias y situaciones que provocaron o agravaron la crisis financiera, dentro de un marco multicausal (Rodil y Menezes, 2011). En este sentido Álvarez y Gonzalez-Parámo (2012) señalan las deficiencias en la regulación y supervisión junto las causas macroeconómicas y microeconómicas, Asimismo, Taylor (2009) considera que la FED mantuvo unos tipos oficiales excesivamente bajos respecto a una regla de política monetaria establecida, habitualmente denominada regla de Taylor. Desde un modelo de Equilibrio General Dinámico y Estocástico (DGSE), Gerali et al (2010) sostienen que el sector bancario influye en mayor medida que la evolución macroeconómica, y lo hace de varias formas, suavizando los shocks de demanda, estimulando los de oferta y condicionando (mediante la inversión) a toda la economía ante pérdidas de capital.

La política de respuesta desarrollada por el BCE también ha sido objeto de estudio,

Papeles de Europa

Vol. 27, Núm. 1 (2014): 77-104

http://dx.doi.org/10.5209/rev_PADE.2014.v27.n1.47013 
Cárdenas, Luís. Análisis de la gestión monetaria del BCE desde un enfoque de endógeno.

entre los recientes análisis que se han realizado sobre esta cuestión cabe destacar a Ayuso y Malo de Molina (2011), Esteve y Prats (2011), Millaruelo y del Río (2013), y Malo de Molina (2013). Todos ellos destacan que la política convencional ha consistido en un drástica reducción de los tipos de interés como consecuencia de la caída de la actividad económica y de un período deflacionario en la Eurozona, ha esta práctica se le encuadra dentro de las medidas convencionales, aunque como se expone posteriormente el BCE intento subió moderadamente el tipo oficial. Además, ante la gravedad de la crisis financiera, deficiencias de diseño y la proximidad de los tipos de interés a cero se han desarrollado otros instrumentos considerados no convencionales.

Las medidas de respuesta se han centrado en mantener la liquidez del sistema y, sobretodo, en el mantenimiento de la masa monetaria mediante el mantenimiento o recuperación del mecanismo de transmisión del sector financiero; como sostiene González-Páramo (2012, p. 90):

[EI] conjunto de medidas estaba dirigido a prestar apoyo al sector bancario en razón del papel central que éste desempeña para el mecanismo de transmisión de la política monetaria en la zona del euro. De hecho, casi el $80 \%$ de las necesidades de financiación de las sociedades no financieras de la zona del euro se satisfacen mediante créditos bancarios (a diferencia de Estados Unidos, donde esta cifra es inferior al 40\%).

Las primeras actuaciones no han tenido el éxito esperado, Corbatón, Molina y Sardá (2011) señalan que las medidas no ha tenido el efecto suficiente como para recomponer el mercado interbancario, como consecuencia la política laxa debe mantenerse hasta que se evite la posibilidad de estrangulamientos de liquidez. Desde un punto de vista histórico, Eichengreen y Temin (2010) comparan las restricciones de política monetaria en la actualidad con las ideas que permitieron el establecimiento y mantenimiento del patrón oro; siendo herederas de este sistema surgen los mismos problemas, una sensación de confianza ante períodos de crecimiento y graves desequilibrios ante fases de recesión o crisis. Desde un enfoque diferente De la Pola (2012), realiza un análisis crítico del BCE como heredero del Bundesbank y su excesiva vigilancia de la inflación que fomenta medidas potencialmente recesionistas.

Los diversos autores mantienen un patrón de pensamiento común (bajo los fundamentos previamente señalados), la política monetaria que puede desarrollar el BCE es limitada y en gran medida las políticas que deberían desarrollarse se encuentran sometidas a factores externos, aún así su actuación fue relativamente rápida, con la introducción de novedosos elementos que permitieron mitigar los efectos de la crisis financiera, de forma semejante a la gestión seguida por los principales bancos centrales. Dada la situación excepcional de crisis de deuda soberana en la Eurozona a partir del 2012, la política monetaria incluye una mayor actuación en los mercados secundarios de deuda soberana en el marco de unas medidas no convencionales, que ha surtido efecto relativamente $\mathrm{y}$, por lo tanto, han sido beneficiosas para el conjunto de la actividad económica.

Esta línea de pensamiento es coherente con el denominado Nuevo Consenso Macroeconómico (NCM), siguiendo a Arestis (2011), esta teoría sostiene como puntos centrales los siguientes: 1) La economía de mercado es estable, y la política fiscal discrecional puede crear distorsiones del equilibrio de mercado, 2) Los mercados financieros juzgan correctamente utilizando la información disponible, pero 
Cárdenas, Luís. Análisis de la gestión monetaria del BCE desde un enfoque de endógeno.

existen rigideces e información asimétrica en el corto plazo; 3) la política monetaria determina la inflación, en el largo plazo sólo puede actuar garantizando la estabilidad de precios. Siendo este su principal objetivo debe ser desarrollado por expertos independientes de las autoridades públicas, y guiarse por reglas que aporten credibilidad (como el inflation-target, IT) 4) la política fiscal no es un instrumento útil y debe centrarse en mantener el presupuesto equilibrado, i.e., la consolidación fiscal. 5) La actividad económica fluctúa sobre la NAIRU (Non-Accelerating-Inflation-Rateof-Unemployment) siendo un fenómeno del lado de la oferta y no existiendo relación entre inflación y desempleo en el largo plazo.

Comparando esta definición de NCM con la revisión de la literatura reciente previamente desarrollada y los principios de estrategia monetaria del BCE es posible concluir que de facto o de iure se ajustan perfectamente a esta corriente de pensamiento. Por lo tanto, todos los análisis realizados sobre la gestión monetaria del BCE y el resto de bancos centrales durante la crisis financiera están condicionados por esta visión, aunque existen discrepancias sobre algunos puntos como el pilar monetario (de tradición cuantitativa) existe un cuerpo de análisis común que limita las posibilidades de actuación.

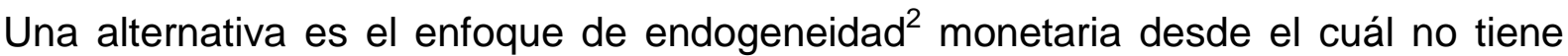
sentido el objetivo cuantitativo de la masa monetaria, pues el banco central no dispone de su pleno control, ni la relación entre dinero e ingreso es estable (Fontana y Palacio-Vera, 2004). El enfoque endógeno sostiene que nivel y la evolución de la masa monetaria se encuentra determinada por la demanda de créditos del sector privado al sector bancario. Sus principales características son: 1) el dinero es indisociable de la producción, 2) la relación con los precios es de causalidad inversa, i.e., la producción y precios determinan la masa monetaria, 3) la inversión determina los ahorros, los créditos crean depósitos, y los depósitos crean reservas $\left.{ }^{3}, 4\right)$ las perturbaciones financieras tienen efectos en el largo y en el corto plazo.

Esta forma de análisis monetario tiene consecuencias en las implicaciones de política monetaria, siguiendo a Arestis y Sawyer $(2002,2006)$ la centralidad de la preferencia por la liquidez vuelve a otorgar un papel fundamental a la demanda agregada, de tal forma que la política fiscal es necesaria para realizar medidas expansivas de la producción y la actividad económica. En la presente investigación se va a optar por utilizar este marco conceptual para analizar la política monetaria.

\section{LA POLÍTICA MONETARIA DEL BCE}

\subsection{Consideraciones previas}

El Banco Central Europeo (BCE) presenta una serie de peculiaridades en su diseño, a diferencia de otros bancos centrales como la Reserva Federal (FED) o el Banco de Inglaterra (BoE), el BCE es el banco central de 17 países diferentes, que se forma mediante un proceso evolutivo de integración financiera y monetaria dentro del Sistema Europeo de Bancos Centrales (SEBC). El cuál se inició el 1 de Julio de 1990 y concluyo con la implantación plena de la Unión Económica y Monetaria

\footnotetext{
${ }^{2}$ Dentro de este enfoque existe la conocida controversia entre horizontalistas y estructuralistas, aunque no se ha llegado a una síntesis las investigaciones actuales combinan aspectos de ambas corrientes.

${ }^{3}$ La expresión más habitual es «Loans create Deposits and Deposits makes Reserves»
} 
Cárdenas, Luís. Análisis de la gestión monetaria del BCE desde un enfoque de endógeno.

(UEM) el 1 de Enero de 1999.

La influencia que tiene este origen tardío respecto al resto de grandes bancos centrales se refleja en dos aspectos: a) la política monetaria y, b) la independencia del Consejo de Gobierno (Governing Council) ${ }^{4}$. Respecto a la estrategia de política monetaria, otorga la centralidad de sus objetivos a la estabilidad de precios, de tal forma, que la inflación media en la eurozona medida según el Índice de Precios al Consumo Armonizado (IPCA o HICP) sea del 2 por ciento en el largo plazo y en su entorno coyunturalmente, es decir, un enfoque basado en reglas de respuesta en función del comportamiento de los precios ${ }^{5}$. Esto supone una diferencia sustancial en relación a otros bancos centrales como la FED, el Banco de Inglaterra o el Banco de Japón (BoJ), ya que todos ellos han formado parte (o forman) de las herramientas de política económica ostentada por los gobiernos nacionales.

Esta cuestión guarda relación con el segundo aspecto, la explicita independencia ${ }^{6}$ del BCE, mientras que la mayoría de bancos centrales ha seguido un proceso de desvinculación respecto a los gobiernos nacionales (incluido el Banco de España ${ }^{7}$ ), y mantienen entre sus objetivos el crecimiento y el empleo, el BCE, por el contrario, se crea desvinculado del resto de administraciones públicas, y por lo tanto, de los aspectos de política fiscal y estructura económica.

Para mantener el principal objetivo de estabilidad de precios el Consejo de Gobierno considera dos pilares: el pilar económico, que consiste en el análisis de la evolución de la actividad, de los precios, de las previsiones de coyuntura; y el pilar monetario que se centra en la evolución del sector financiero, los créditos y los principales agregados monetarios ${ }^{8}$.

Se han señalado (Malo de Molina, 2011) las deficiencias de diseño institucional en la creación del BCE que se pusieron de manifiesto con la crisis financiera, pero además por el comportamiento de crecimiento inesperado en la Eurozona, donde los países periféricos mantienen un mayor crecimiento que los propios países centrales

De igual forma, el Pacto de Estabilidad y Crecimiento no sólo limita las posibilidades de la política fiscal sino que impone unas condiciones que no garantizan la convergencia económica, como demuestra que las economías con menor volumen de deuda pública (España e Irlanda) sufrieran en mayor medida la crisis de deuda soberana en 2007, cuando los países centrales como Francia y Alemania superaban el límite establecido del $60 \%$ volumen deuda/PIB. Otra cuestión que se ha desarrollado a raíz de la crisis financiera es la necesidad de una institución que permita gestionar las crisis de liquidez y financiación de los Estados miembros con la suficiente capacidad y velocidad de actuación, en este aspecto se ha desarrollado el Mecanismo Europeo de Estabilidad (MEDE), que es el heredero de la Facilidad

\footnotetext{
${ }^{4}$ Formado por los seis miembros del Consejo Ejecutivo y los gobernadores de los respectivos bancos centrales nacionales.

${ }^{5}$ Véase Taylor, J. B. (1993), sobre la cuestión de las reglas de política monetaria

${ }^{6}$ Dreher, A., Sturm, J.-E. y Haan, J. D. (2010) sostienen que aparte de por finalización de mandato, en la cesión del cargo de gobernador influyen la situación política y de estabilidad del régimen, los procesos electorales y la evolución de la actividad financiera (créditos/ PIB).

${ }^{7}$ Ley 13/1994, de 1 de junio, de Autonomía del Banco de España.

${ }^{8}$ Este punto suscita críticas tanto entre los neokeynesianos, véase Galí, J. (2010) para una crítica de este enfoque basado en el pilar monetario; tanto como en los postkeynesianos (Fontana y Palacio-Vera, 2004)
} 
Cárdenas, Luís. Análisis de la gestión monetaria del BCE desde un enfoque de endógeno.

\section{Europea de Estabilización Financiera (FEEF) y el Mecanismo Europeo de Estabilización Financiera (MEEF).}

Respecto a la posibilidad de adquisición de bonos públicos en el mercado primario, el Tratado de Funcionamiento de la Unión Europea especifica en su artículo 123\%:

Queda prohibida la autorización de descubiertos o la concesión de cualquier otro tipo de créditos por el Banco Central Europeo y por los bancos centrales de los Estados miembros, (...) en favor de instituciones, órganos u organismos de la Unión, Gobiernos centrales, autoridades regionales o locales u otras autoridades públicas, organismos de Derecho público o empresas públicas de los Estados miembros, así como la adquisición directa a los mismos de instrumentos de deuda por el Banco Central Europeo o los bancos centrales nacionales.

\subsection{Política monetaria convencional}

Los instrumentos de los que dispone el BCE consisten en las operaciones de mercado abierto (Open Market Operations) y las facilidades permanentes. En primer lugar, las operaciones de mercado abierto son las principales herramientas de creación de masa monetaria, estos instrumentos se utilizan de forma regular y periódica. Se componen de de dos tipos de operaciones. Las primeras son las denominadas operaciones principales de financiación u OPF (Main Refinancing Operations, MROs), que consisten en inyecciones de liquidez con vencimiento semanal, las subastas se realizan también cada semana. En segundo lugar, las operaciones de financiación a más largo plazo u OFLP (Longer-Term Refinancing Operations, LTROs), tienen una duración de la financiación que suele ser de tres meses, aunque existen diversas fórmulas; las subasta se realizan con periodicidad mensual.

Los instrumentos discrecionales y, por tanto, sin periodicidad definida son las operaciones de ajuste (Fine-tuning Reverse Operations, FTRO) y las operaciones estructurales (Structural Operations). Las operaciones de ajuste son instrumentos que garantizan la existencia de la liquidez prevista, por lo tanto, se utilizan ante situaciones coyunturales de desviación del nivel esperado, la implantación requiere de una rápida actuación mediante subastas o absorciones, en función de su necesidad de drenaje o inyección de líquidez. Por el contrario, las operaciones estructurales se realizan cuando se quiere modificar sustancialmente (estructuralmente) la situación de líquidez del sistema monetario y financiero. Para realizar estas operaciones, el BCE cuenta con la emisión de certificados de deuda (Issuance of ECB debt certificates), los depósitos a plazo fijo y las permutas o swaps de divisas. Por último, el establecimiento de un sistema de reservas mínimas de caja se convierte en un instrumento para la gestión de la liquidez, una reducción del coeficiente ampliará sensiblemente las disponibilidades bancarias, esta medida también debe entenderse como cambios fundamentales en el sistema monetario y no de contingencia.

En segundo lugar, las facilidades permanentes permiten al BCE mantener los tipos de interés interbancarios próximos al tipo oficial, para marcar esta banda de

\footnotetext{
${ }^{9}$ Artículo 101 en el antiguo TCE, también Asimismo en los Estatutos del BCE se señala en su artículo 21.1: "Con arreglo a lo dispuesto en el artículo 123 del Tratado de Funcionamiento de la Unión Europea, queda prohibida la autorización de descubiertos y la concesión de otro tipo de créditos por parte del BCE o de los bancos centrales nacionales en favor de instituciones, órganos u organismos de la Unión, Gobiernos centrales $[\ldots] "$.
} 
Cárdenas, Luís. Análisis de la gestión monetaria del BCE desde un enfoque de endógeno.

fluctuación dispone de las facilidades permanentes de crédito y depósito. Las de crédito determinan el tipo de interés más alto, es decir, aquél al que presta el banco central, por ello el mercado monetario siempre se encontrará por debajo de este límite. Por otra parte las facilidades de depósito establecen el límite inferior sobre el que fluctuarán los tipos nominales, al ser el que remunera el banco central por realizar depósitos.

Gráfico 1. Evolución de los tipos oficiales internacionales, 1990-2013.

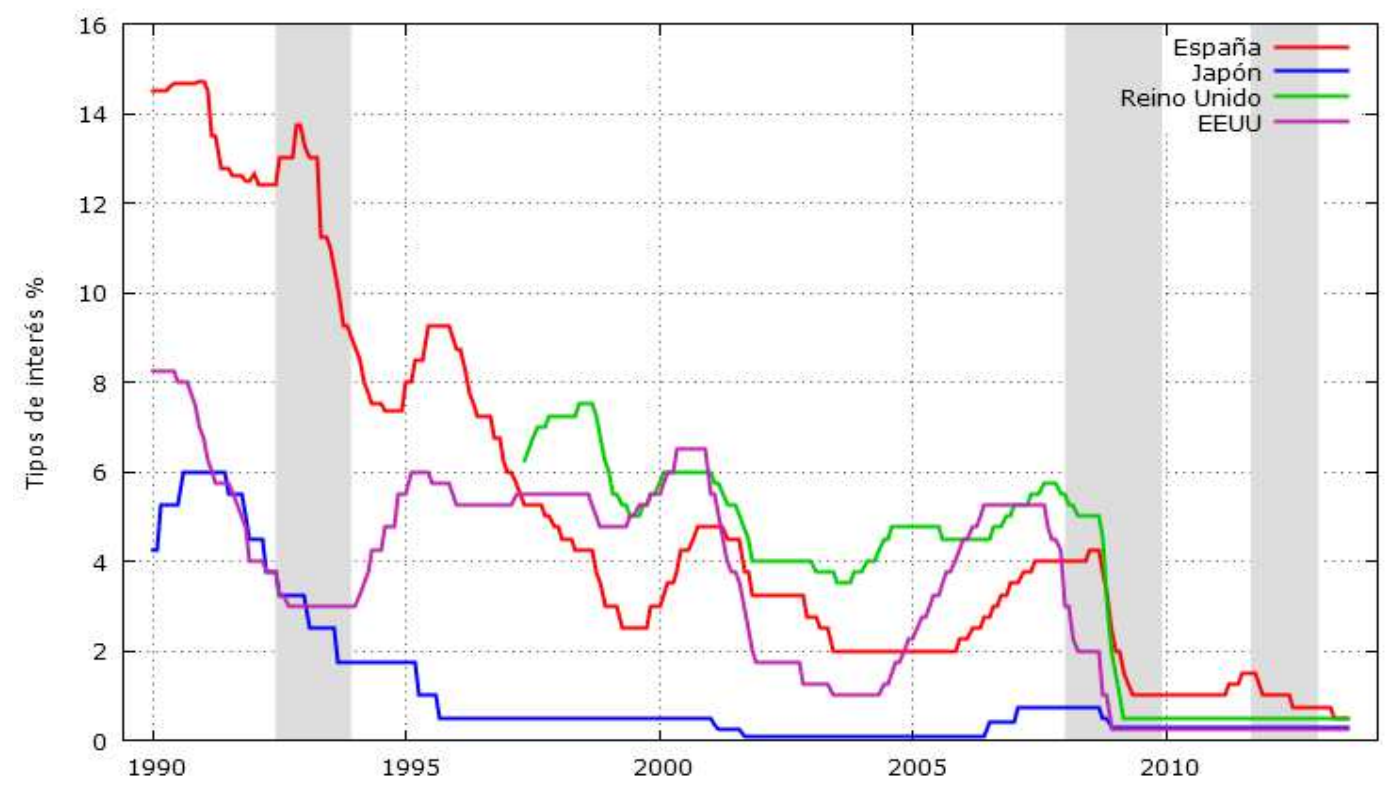

Nota: Las zonas sombreadas indican recesión en España.

Fuente: Elaboración propia a partir de los datos del ํㅡo de Economía y Competitividad (2013).

Como se observa en el Gráfico 1, la evolución en las últimas décadas de los tipos de interés oficiales de los principales bancos centrales (Reserva Federal, (FED), el Banco de Japón (BoJ), el Banco de Inglaterra (BoE) y el BCE a partir de 1999 y previamente el Banco de España (BdE), tiene una tendencia temporal a ir reduciéndose progresivamente. Esta situación se observa claramente en la crisis de los primeros años noventa, durante la cuál los bancos centrales bajaron drásticamente los tipos oficiales, algunos como el BoJ no volverían a subirlos a partir de 1995 como si hicieron la FED y el BdE. El caso español también es paradigmático de esta tendencia a la baja es el español que incluso los mantiene en niveles inferiores a los de la FED y el BoE.

La respuesta convencional a la crisis y la "Gran Recesión» ha consistido en una nueva reducción del tipo oficial, incluso el BoJ ha aproximado aún más sus tipos a cero, creando un nuevo escenario monetario internacional sin posibilidad de mayores reducciones.

En el caso del BCE, se suelen distinguir 6 etapas en su estrategia monetaria: 1) la introducción del euro entre los años 98 y 99,2) una subida de tipos del 99 a los años $2000,3)$ un ajuste a la baja (2001-03), 4) el mantenimiento del tipo constante desde el 2003 hasta el 2005, 5) modificación del tipo de subasta (acomodaticia) entre el 2005 y el 2008, 6) actuación del BCE frente a la crisis financiera (a partir del 2008). 
Cárdenas, Luís. Análisis de la gestión monetaria del BCE desde un enfoque de endógeno.

En este trabajo se opta por una definición diferente de las fases en la gestión de los tipos oficiales (véase gráfico 2); se distinguen dos períodos de subidas y dos períodos de bajadas. La primera fase consistió en un aumento y posterior bajada del tipo oficial durante los años (1999-2002), esta reducción se defiende en función del desarrollo macroeconómico, en especial, por el menor crecimiento de los países centrales frente a los países periféricos, el mayor peso que los primeros requería un estímulo a riesgo de efectos deflacionistas en la Eurozona. La segunda etapa dura hasta el 2006 sin modificaciones en los tipos de interés. A partir del 2006, se produce una subida escalonada hasta el $4 \%$, desde donde cae drásticamente hasta el $1 \%$. Aunque (como se observa en el gráfico 1) esta medida se produjo con posterioridad al inicio de la recesión en el caso español, siendo el BCE el último de los bancos estudiados en reaccionar y manteniendo un tipo superior al resto.

Gráfico 2. Gestión de los tipos en la Eurozona, 1999-2013.

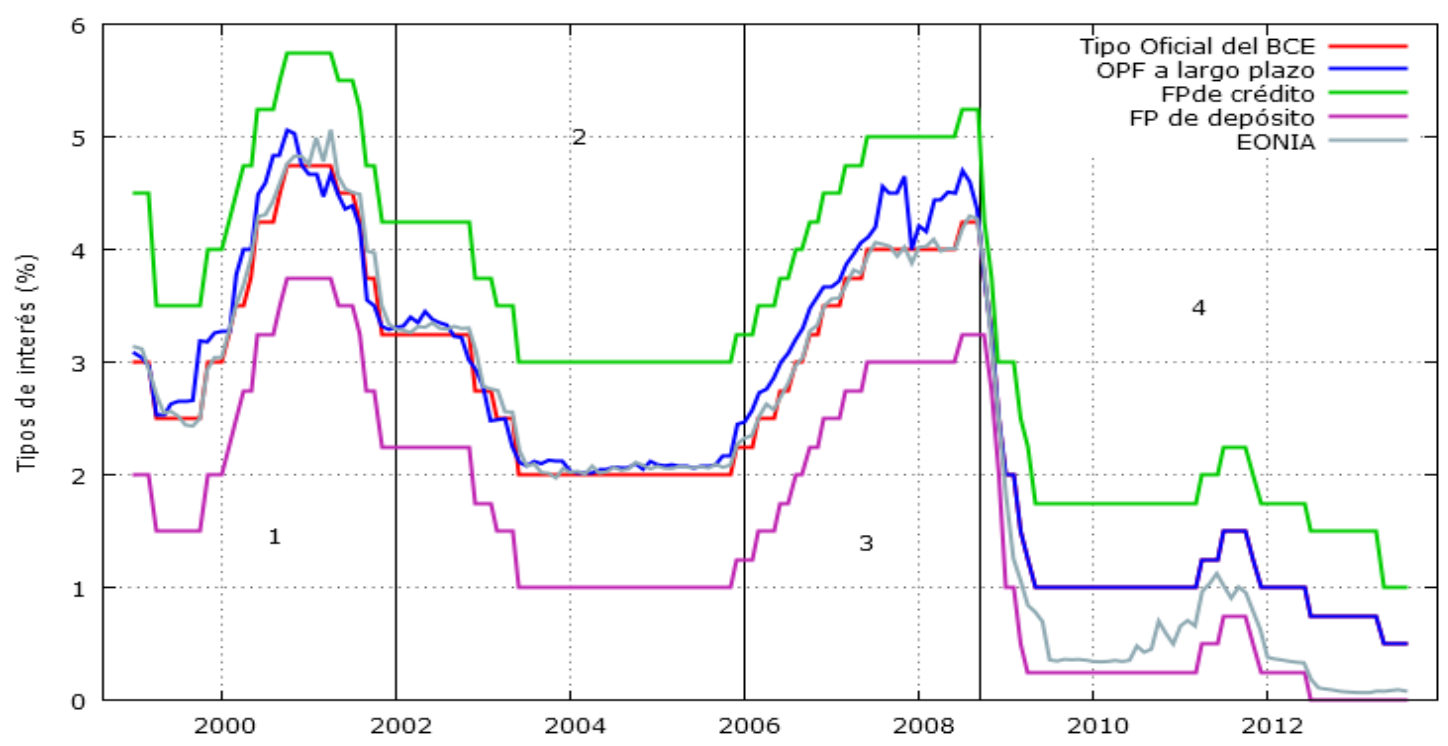

Fuente: elaboración propia.

El papel jugado por el prestamista de última instancia a consistido en la inyección de liquidez, el principal problema es que no ha estimulado el mercado monetario, según el Informe de estabilidad financiera ${ }^{10}$,

[Los mercados monetarios] han seguido caracterizándose por su débil actividad. La permanencia de la desconfianza debida a la crisis de la deuda soberana ha propiciado que el volumen de actividad de los mercados interbancarios haya seguido registrando niveles significativamente inferiores a los de los años previos.

Esta situación de tipos internacionales bajos y facilidades de obtención de líquidez (zero lower bound, $Z L B$ ) es considerado como un escenario donde se pueden crear una serie de problemas a largo plazo; en esta línea Hernando, Llopis y Vallés (2012) señalan algunos de estos aspectos problemáticos de distorsiones de mercado y de incentivos. Se reducen los incentivos a cambios sustanciales (y potencialmente problemáticos), es el caso de las entidades bancarias en el saneamiento de sus balances, en el caso de los Estados miembros de la UME a la consolidación fiscal (por la reducción del valor de la deuda pública en situaciones inflacionistas) que suscribieron en el Pacto de Estabilidad y Crecimiento. Otro efecto es la reducción del número de instituciones financieras y monetarias, i.e., una mayor concentración,

\footnotetext{
${ }^{10}$ Informe de estabilidad financiera. Noviembre, 2012. Banco de España.
} 
Cárdenas, Luís. Análisis de la gestión monetaria del BCE desde un enfoque de endógeno.

como consecuencia de una reducción de los márgenes de intermediación de los bancos, efectos spillover sobre las economías emergentes, lo cuál implica un mayor volumen de flujos de capital hacía las economías emergentes y de mayor crecimiento (como los denominados BRICS).

A pesar de estas consideraciones, la principal hipótesis en la situación de tipos de interés bajos es la existencia de una trampa de la liquidez, idea central en el pensamiento de Keynes, y que como señala Paul Krugman (1998) se considero una teoría poco realista de producirse en la práctica,. En ese mismo trabajo Krugman compara la trampa de líquidez que pudo producirse durante la Gran Depresión estadounidense con la evolución de Japón en la década de los noventa (1987-1997). Sus conclusiones están íntimamente relacionadas con la situación del euro tras la crisis financiera: Krugman (1998, p. 183)

Europe faces Japanese-style demographics; could a liquidity trap happen to the European Monetary Union? Economists now know that the liquidity trap is not a historical myth: it can and does really happen sometimes, and we had better try to understand it.

El otro aspecto clave para comprender la política convencional es el mecanismo de transmisión de la política monetaria o monetary transmission mechanism (MTM), el cuál se define como la forma en que las variaciones de la masa monetaria nominal o de la tasa de interés nominal a corto plazo afectan a variables reales tales como la producción agregada y el empleo, siendo el resultado de la aplicación de una política determinada.

El BCE mantiene una estructura de transmisión centrada en la estabilidad de precios, los tipos de interés oficiales determinan los del mercado monetario y conjuntamente con las expectativas los volúmenes de dinero, crédito, precios de los activos, tipos del interbancario y el tipo de cambio. Lo cuál influye en la oferta y demanda de bienes y factores productivos que va determinar la evolución de los precios. Siendo considerados exógenos la evolución y capitalización financiera, cambios de políticas fiscales o los precios de las materias primas.

Tabla 1. Enfoques del Mecanismo de Transmisión.

\begin{tabular}{|c|c|}
\hline Enfoque & Proceso \\
\hline Keynesianismo Tradicional & $M \downarrow \rightarrow i T \rightarrow Z \downarrow \rightarrow Y L$ \\
\hline Canal del Tipo de Cambio & $M \downarrow \rightarrow i \uparrow \rightarrow E \uparrow \rightarrow N X \downarrow \rightarrow Y \downarrow$ \\
\hline Efecto en el Precio de otros Bienes & $M \downarrow \rightarrow p_{z} \downarrow \rightarrow q \downarrow \rightarrow I \downarrow \rightarrow Y \downarrow$ \\
\hline Canal del Crédito & $M \downarrow \rightarrow$ bonkdeposits $\downarrow \rightarrow$ bonklowns $\downarrow \rightarrow I \downarrow \rightarrow Y \downarrow$ \\
\hline \multicolumn{2}{|c|}{$\begin{array}{l}\text { Nota: Las variables son respectivamente: masa monetaria (M), tipo de interés (i), inversión } \\
\text { (I), producción (Y), tipo de cambio (E), exportaciones netas (NX), el precio de las acciones } \\
\text { (Pe), la valoración de mercado de las empresas medida como la q de Tobin (q) y los } \\
\text { depósitos (bank deposits) y préstamos bancarios (bank loans). } \\
\text { Fuente: elaboración propia a partir de Mishkin (1995) }\end{array}$} \\
\hline
\end{tabular}

El MTM no esta exento de diversas interpretaciones por parte de la teoría económica, es habitual diferenciar entre: el Keynesianismo tradicional o del tipo de interés (efecto renta, riqueza, etc...) el canal del tipo de cambio con modificaciones en la balanza comercial, el efecto en el precio de otros bienes (q de Tobin), y el canal crediticio del sector bancario. Sobre estos MTM influyen al menos dos efectos, las expectativas y el efecto desbordamiento (spillover). En la Tabla 1 se sintetizan los 
Cárdenas, Luís. Análisis de la gestión monetaria del BCE desde un enfoque de endógeno.

distintos enfoques.

Siguiendo a Ceccioni y Neri (2011), se consideraba que el patrón de comportamiento del MTM se caracterizaba por una homogeneidad entre países, teniendo la política monetaria efectos a corto plazo en la producción, siendo su principal canal el del tipo de interés; además se relegaba a las fricciones financieras a un rol secundario. Estos autores argumentan que se han producido cambios en este patrón, de tal forma que ahora es el sector privado el que determina en mayor medida las fluctuaciones de la producción y el empleo causadas por un shock monetario; mientras que la reducción de la volatilidad macroeconómica no es atribuible a la política monetaria, que tiene efecto en el control de precios. Se puede razonar que este último resultado es consecuencia de la fijación de la estabilidad de precios como principal objetivo.

Gerali et al (2010) sostienen que los shocks en el sector financiero explican en gran medida que el resto de condiciones macroeconómicas la caída de la producción en el 2008, especialmente en una situación de quiebra bancaria que reduce el capital financiero y determina la inversión. En resumen, la oferta y demanda de financiación crediticia es una de las principales explicaciones de las fluctuaciones económicas.

Para ayudar a resolver los problemas del mecanismo de transmisión creditício, los bancos centrales han respondido con la denominada «relajación cuantitativa» ${ }^{11}$ (Quantitative Easing), que consiste en una política de expansión del balance del banco central mediante un aumento de pasivos (inyecciones monetarias), pero sin modificar sustancialmente la composición de sus activos, es decir, sin modificaciones en su política de adquisiciones de activos financieros. Si este cambio de orientación en la práctica de adquisiciones se produce se denomina «relajación cualitativa» (Qualitative Easing), es decir, no sólo aumenta su pasivo sino que además realiza modificaciones en el tipo de activo. De igual forma ha actuado la Reserva Federal Estadounidense (FED), como sostiene Ben Bernanke (2011):

To help stabilize the financial system and facilitate the flow of credit to households and businesses, the Federal Reserve responded to the dislocations in funding and securitization markets by dramatically increasing the amount of term funding that it provided to banks, establishing new lending facilities for nonbanks, and providing funding to support the operation of key markets.

\subsection{Política monetaria no convencional}

Con el objetivo de corregir este estancamiento monetario y haber alcanzado el límite de los instrumentos tradicionales, el BCE, en línea con los principales bancos centrales, ha desarrollado una serie de medidas denominadas «no convencionales», aunque como señala Lenza, et al (2010), no es sencillo etiquetarlas en las «relajaciones cuantitativas» o en las «relajaciones cualitativas».

La literatura sobre la cuestión se ha desarrollado rápidamente, Joyce, Tong y Woods (2011) consideran que el mecanismo mediante el que actúan estas políticas es el canal de precios de los activos y los créditos bancarios, aplicando el Teorema de Wallace. En esta línea, Bowdler y Radia (2012) realizan una síntesis al respecto

\footnotetext{
11 También denominada flexibilización o facilidad cuantitativa, expansión monetaria cuantitativa (EMC), etcétera.
} 
Cárdenas, Luís. Análisis de la gestión monetaria del BCE desde un enfoque de endógeno.

distinguiendo entre las habituales (convencionales) medidas «no convencionales» y las no convencionales políticas «no convencionales». Es decir, una serie de prácticas eran consideradas hasta la fecha como heterodoxas, pero su introducción por parte de los principales bancos las ha convertido en una práctica habitual, es el caso de los incrementos de balance y las adquisiciones de activos financieros (en resumen las QE). Estos autores señalan que el mecanismo por el que actuará esta política es la reestructuración de las carteras de los agentes, aportando que en ese nuevo equilibrio (aunque a largo plazo para evitar la equivalencia ricardiana estricta) de inversiones debe producirse las no convencionales (orientación de las expectativas, mantenimiento de la liquidez, y fomento del crédito).

Tabla 2. Medidas «no convencionales» adoptadas por el BCE.

\begin{tabular}{ll}
\hline \multicolumn{1}{c}{ Tipo } & \multicolumn{1}{c}{ Procedimiento } \\
\hline OPF & Tipo fijo y adjudicación plena en la subasta \\
\hline LTRO & Ampliación de plazos: 3, 6, 12 meses y VLTRO: 3 años \\
\hline SWAPS & Acuerdos de permuta de divisas con la FED y el Banco Nacional Suizo \\
\hline CBPP, SMP y OMT & Compra títulos, bonos bancarios y soberanos en el mdo. secundario \\
\hline Coeficiente de Caja & Reducción del coeficiente de reservas exigidas al 1\% \\
\hline
\end{tabular}

Fuente: elaboración propia.

La implantación de estos instrumentos (Tabla 2) ha consistido en cinco grandes bloques:

1) La sustitución de una Subasta Discriminatoria, consistente en una subasta al primer precio, ya que las unidades se adjudicarían a las pujas más altas y se pagaría un precio igual a su puja. Por una Subasta de Precio Uniforme que se asemejan a la subasta al segundo precio, es decir, se adjudicaría a las pujas más altas pero todos pagarían el mismo precio, el cual sería igual a la puja más alta de las rechazadas o la más baja de las aceptadas.

Es importante que el precio se determine de esta manera para que mantenga las propiedades de la subasta al segundo precio ya que, así, el precio finalmente pagado será independiente de las pujas presentadas por cualquiera de los ganadores.

Además se realiza una adjudicación plena, consistente en otorgar toda la cantidad demandante, este sistema es inevitable si se desea evitar el problema de que se solicite excesivamente en cantidad porque el pujador quiere garantizar que recibirá una cantidad mínima y teme que el resto de pujadores inflen su demanda en función del último prorrateo, de tal forma que la cantidad siempre sea muy superior a la realmente deseada.

2) El incremento de los plazos en las operaciones a más largo plazo, aumentado a 6 y 12 meses, e incluso llegando hasta a los 3 años en las denominadas operaciones de muy largo plazo (Very Longer-Term Refinancing Operations, VLTROs).

3) El BCE alcanzó una serie de acuerdos de permuta de divisas (swaps) extranjeras, especialmente, por dólares con la Reserva Federal para facilitar el cumplimiento de los compromisos de los bancos europeos en esta moneda. 
Cárdenas, Luís. Análisis de la gestión monetaria del BCE desde un enfoque de endógeno.

4) La actuación en los mercados secundarios de valores mediante la compra de títulos y bonos, especialmente con la finalidad de reducir la presión sobre la deuda soberana en algunos países, se ha desarrollado por medio de distintos programas, primero el Programa de compra cubierta de bonos que han consistido en el Covered Bond Purchase Programme y en el Covered Bond Purchase Programme 2 (CBPP y CBPP2) que de forma conjunta han adquirido activos bancarios garantizados por un valor nominal de 100 mil millones de euros $(60 \mathrm{~mm}$ y $40 \mathrm{~mm}$ respectivamente). Posteriormente, en mayo del 2010, se aplicó el Programa para el Mercado de Valores (Securities Markets Programme, SMP), que consistía en la compra de bonos soberanos en los mercados secundarios.

Esta actuación se enmarca dentro de la crisis de deuda soberana que elevó significativamente la remuneración de la deuda pública y creó importantes problemas de financiación. Como se observa en la Tabla 3, los resultados del programa una vez finalizado muestran que el principal beneficiario de este programa ha sido Italia que colocado hasta $102,8 \mathrm{~mm}$ de $€$, un $47 \%$ del total de la operación, los bonos españoles alcanzan los $44 \mathrm{~mm}$, el caso de Grecia es significativo a parte de por su menor tamaño por ser el primero sobre el que se actuó y sus bonos son los más próximos al vencimiento.

Tabla 3. Resumen del Programa para el Mercado de Valores.

\begin{tabular}{lccc}
\hline \multirow{2}{*}{ País Emisor } & \multicolumn{2}{c}{ Importes Pendientes } & $\begin{array}{c}\text { Plazo de vencimiento } \\
\text { medio (en años) }\end{array}$ \\
\cline { 2 - 4 } & Valor nominal $(\mathbf{m m} \boldsymbol{€})$ & Valor en libros $(\mathbf{m m} \boldsymbol{€})$ & 4,60 \\
\hline Irlanda & 14,20 & 13,60 & 3,60 \\
\hline Grecia & 33,90 & 30,80 & 4,10 \\
\hline España & 44,30 & 43,70 & 4,50 \\
\hline Italia & 102,80 & 99,00 & 3,90 \\
\hline Portugal & 22,80 & 21,60 & 4,30 \\
\hline Total & 218,00 & 208,70 &
\end{tabular}

Fuente: Banco Central Europeo, 2013.

Como continuación del SMP se instrumenta a partir de Septiembre de 2012 el programa de Operaciones Monetarias de Compraventa (Outright Monetary Transactions, OMT), con el objeto de garantizar la estabilidad del euro, se realizan compras en los mercados secundarios de deuda pública, sin límites de cantidad pero sujeto a un programa completo de reforma macroeconómica en el marco de la creación del Mecanismo de Estabilidad Europeo (MEDE).

5) Respecto a los requisitos de reservas exigidos, se redujo al $1 \%$ el coeficiente de caja que se había situado tradicionalmente en el $2 \%$, en un intento de dotar aún de mayor liquidez al sistema monetario. Junto a estas medidas también se amplió la lista de activos susceptibles de ser considerados como garantías en las operaciones con el BCE otorgando mayor flexibilidad a los operadores de contrapartidas, llegando incluso a plantearse algún tipo de instrumento que permita una financiación más directa a las empresas. 
Cárdenas, Luís. Análisis de la gestión monetaria del BCE desde un enfoque de endógeno.

La declaración ${ }^{12}$ de Mario Draghi, Presidente del BCE, «haremos todo lo necesario para salvar el euro» (do whatever it takes to save the euro), es un buen ejemplo de la necesidad de creación de expectativas mediante el establecimiento de estas políticas «no convencionales», posteriormente desarrollaba esa frase:

Whatever it takes" means two things: it means the list of measures, all the measures that are required, and it means that their size ought to be adequate to reach their objectives. And so the various committees will now review the various non-standard policy options. And then we will meet and we will decide what to do.

Finalmente, sobre la evidencia empírica disponible (Millaruelo y Del Río, 2013), siendo aún poco abundante se pueden distinguir dos líneas de argumentación, los que consideran que la política realizada esta siendo efectiva y los que defienden que es insuficiente.

Entre el primer grupo se encuentran los trabajos de Giannone et al. $(2010,2012)$ y Lenza et al. (2010), estos autores realizan principalmente estimaciones desde una metodología de modelos Bayesianos de Vectores Autorregresivos (B-VAR), el planteamiento consiste en realizar predicciones a partir del año 2007 en dos posibles escenarios, con la implantación de medidas no convencionales y sin ellas, llegando al resultado de que las medidas no convencionales han apoyado al funcionamiento del mercado financiero, al MTM y con ello la política monetaria ha tenido un efecto beneficioso sobre la economía real, reforzando así la actividad macroeconómica y el empleo de manera moderada pero significativa. En este grupo también se encuentra el estudio de Joyce, Tong y Woods (2011) que en el caso británico tienen un efecto positivo en la producción (entre un 1,5 y un 2 por ciento) y en la inflación $(0,75-$ $1,5 \%)$.

Por el contrario, Corbatón, Molina y Sardá (2011) sostienen que el mercado interbancario no muestra signos de mejoría, aunque retirar estos estímulos pondrían en una situación aún más complicado. De la Pola (2012), considera que las medidas de política aplicadas están excesivamente restringidas por el miedo a la inflación, lo cuál limita el potencial efecto expansivo.

\section{HECHOS ESTILIZADOS}

\subsection{Las variables monetarias}

En este apartado se desarrolla una descripción de las principales variables de política monetaria y la evolución de los agregados, en este aspecto se puede concluir que la decisión del BCE de estimular la oferta mediante la adopción de políticas «no convencionales» le permiten mantener la líquidez necesaria en la economía ha permitido a las entidades cumplir con sus compromisos, pudiendo evitar las quiebras bancarias, los banks run, el estrangulamiento del crédito y la deflación; teniendo cualquiera de estos escenarios efectos contractivos de la actividad económica que agravaría la recesión tanto de la Eurozona como de España, aún así no se ha producido una reactivación del mercado interbancario y el acceso a los mercados crediticios.

\footnotetext{
12 Ruedas de prensa de Mario Draghi los días 26 de Julio y 2 de Agosto del 2012 respectivamente (http://www.ecb.europa.eu/press/pressconf/2012/html/is120802.en.html)
} 
Cárdenas, Luís. Análisis de la gestión monetaria del BCE desde un enfoque de endógeno.

En el Gráfico 3 se pueden observar las principales variables monetarias, concretamente la variación de la oferta monetaria mantenía un crecimiento estable desde el año 2000, llegando la M3 a aproximarse a los $1000 \mathrm{~mm}$ de $€$. A partir del año 2008 sufrió una fuerte caída, siendo mayor la reducción del M3 que el la caída del agregado M2. El comportamiento del agregado monetario más desagregado, el M1, que venía cayendo desde el 2006, se elevó rápidamente hasta el año 2010 donde a partir de esa fecha siguió la tendencia general de caída.

Gráfico 3 Evolución de la oferta monetaria. Eurozona, 2013.

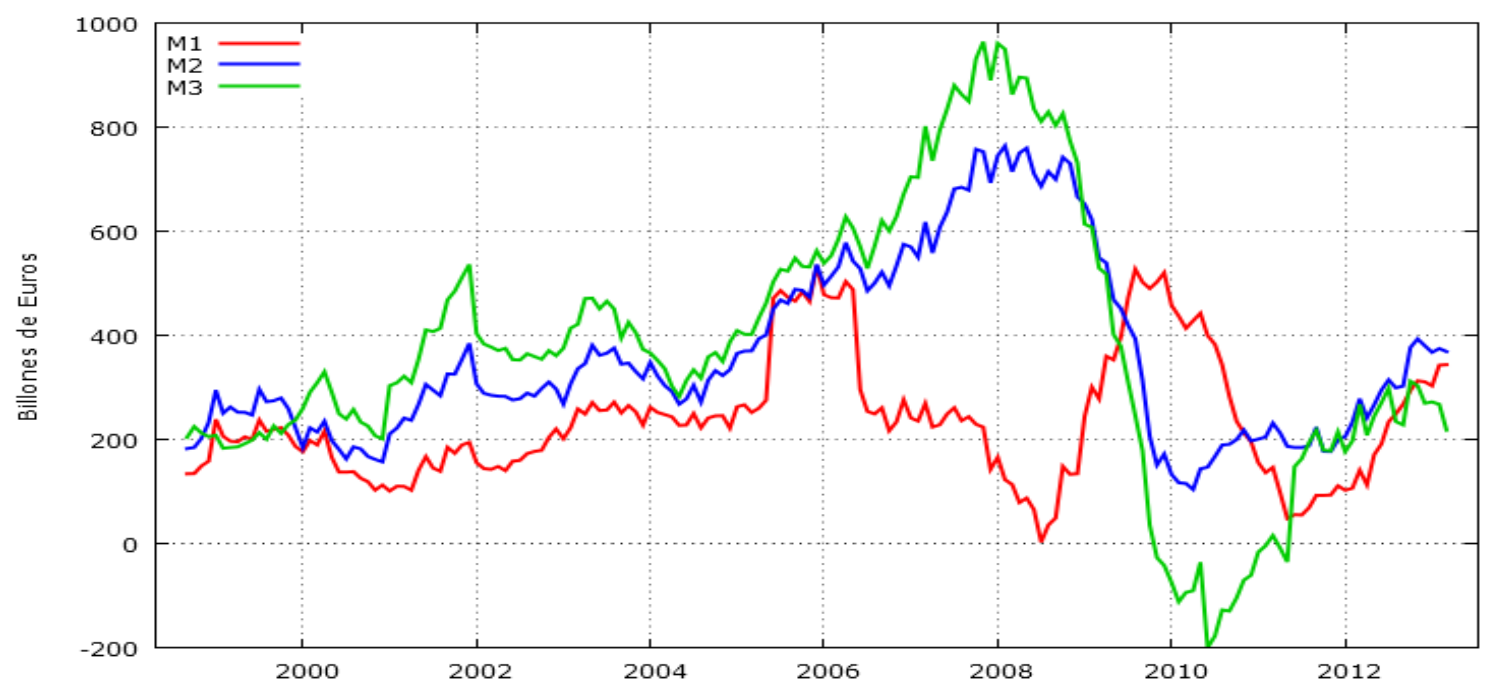

Nota: Resultados obtenidos a partir de las diferencias estacionales de las series de la base da datos BDSICE.

Fuente: elaboración propia a partir de los datos del Mํ de Economía y Competitividad.

En esta línea, un análisis del comportamiento cíclico de las variables los datos de la Tabla 4 muestran una mayor volatilidad relativa de la M3 y una ligeramente menor de la M2, por el contrario los datos en logaritmos la M2 es ligeramente más volátil que la M3, aunque ambas variables pasan a ser relativamente más rígida. Respecto a la correlación cruzada, los resultados indican un comportamiento contracíclico, siendo claramente negativas hacia M1. Manteniendo una correlación sensiblemente más alta entre los períodos pasados y futuros, esto muestra el funcionamiento de transmisión de la masa monetaria y el diferente comportamiento entre las variables.

Tabla 4: Dinámica Cíclica. Eurozona, 2013.

\begin{tabular}{cccccccc}
\hline \multirow{2}{*}{ Variable } & \multirow{2}{*}{ Volatilidad } & \multirow{2}{*}{$\begin{array}{c}\text { Volatilidad } \\
\text { Relativa }\end{array}$} & \multicolumn{5}{c}{ Correlación Cruzada sobre M1 y Log (M1) } \\
\cline { 5 - 8 } & & $X_{t+2}$ & $X_{t+1}$ & $X_{t}$ & $X_{t-1}$ & $X_{t-2}$ \\
\hline M1 & 63,90 & 1 & 0,6258 & 0,7872 & 1,00 & 0,7872 & 0,6258 \\
\hline M2 & 62,49 & 0,978 & $-0,2592$ & $-0,2043$ & $-0,0643$ & $-0,2645$ & $-0,4332$ \\
\hline M3 & 94,19 & 1,474 & $-0,3372$ & $-0,3387$ & $-0,3218$ & $-0,4521$ & $-0,5667$ \\
\hline Log (M1) & 0,369 & 1 & 0,5012 & 0,7183 & 1,00 & 0,7183 & 0,5012 \\
\hline Log (M2) & 0,299 & 0,810 & $-0,9060$ & $-0,0587$ & 0,1644 & $-0,0750$ & $-0,3137$ \\
\hline Log (M3) & 0,289 & 0,783 & $-0,2967$ & $-0,2346$ & $-0,1596$ & $-0,3037$ & $-0,4603$ \\
\hline
\end{tabular}

Nota: Propiedades de los segundos momentos de los datos filtrados por HP.

Fuente: Elaboración propia a partir de los datos del ํㅡde Economía y Competitividad 
Cárdenas, Luís. Análisis de la gestión monetaria del BCE desde un enfoque de endógeno.

Respecto a los cambios de tendencia en el largo plazo tanto en el caso concreto de España y de la Eurozona (Gráfico 4), la dinámica que muestran las variables es un claro crecimiento hasta el año 2008, a partir del cual se inicia un estancamiento en la variación de tendencia que prosigue con una reducción de todos los agregados, en el caso de España continua a partir de la segunda etapa en recesión que se inicia en el 2012, por el contrario en la Eurozona esta situación no se produce.

Gráfico 4. Tendencias de la oferta monetaria.

Evolución en España

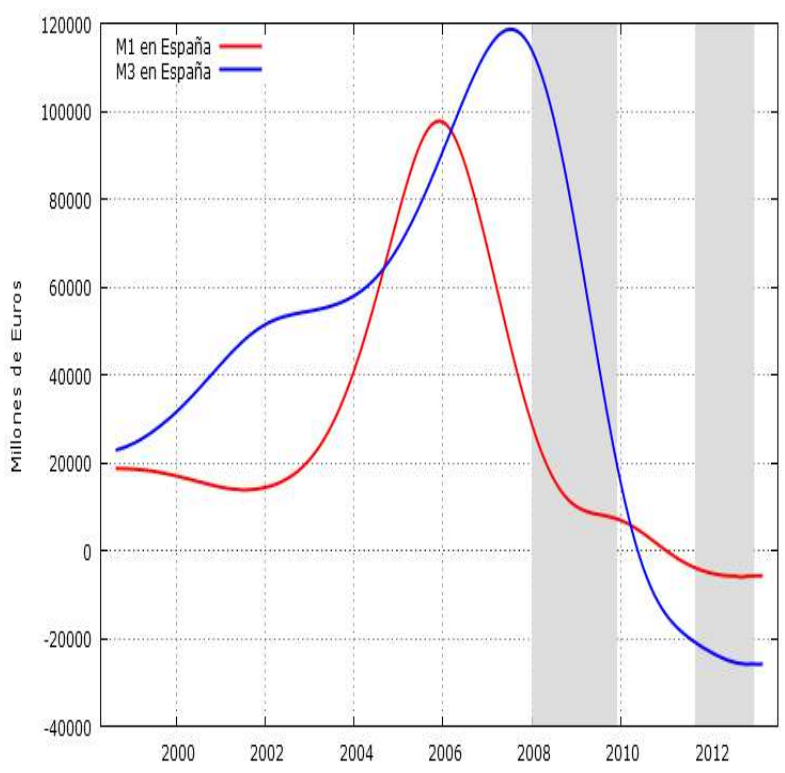

Tasa de Variación en España

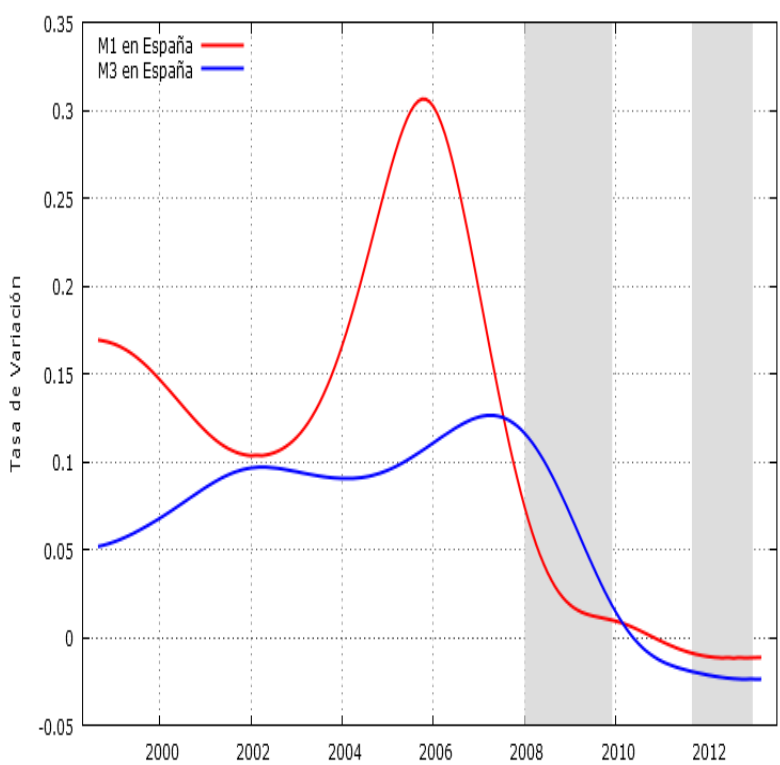

Evolución en la Eurozona

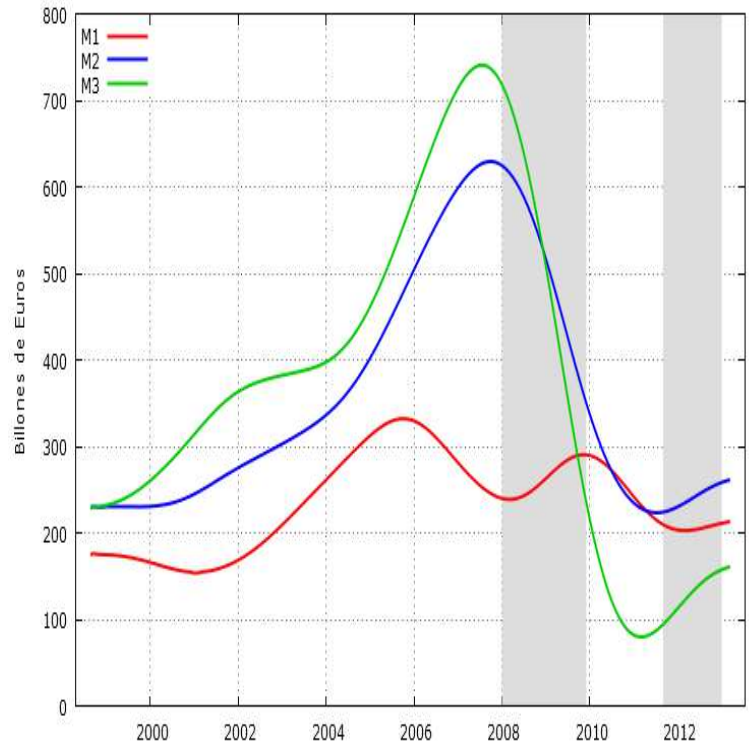

Tasa de Variación en la Eurozona

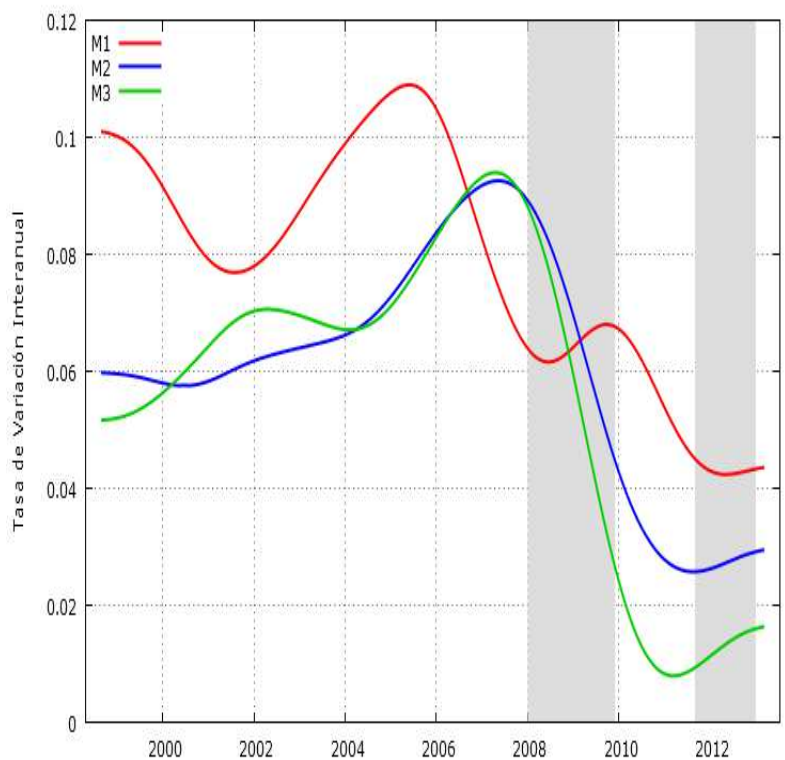

Nota: La tendencia se ha obtenido mediante la aplicación del Filtro Hodrick-Prescott, estableciendo un lambda de 14400 al ser series mensuales. Las zonas sombreadas indican recesión en España.

Fuente: elaboración propia a partir de los datos del ํo de Economía y Competitividad y del BCE.

Las tasas de variación tendenciales muestran que en ambos casos hay un fuerte caída de la masa monetaria, pero mientras que en la Eurozona se mantiene en 
Cárdenas, Luís. Análisis de la gestión monetaria del BCE desde un enfoque de endógeno.

términos positivos en el caso español el volumen monetario continúa con la caída hasta alcanzar posiciones negativas, sin observarse la recuperación que produce en la Eurozona. Según los resultados de la Tabla 5 la evolución temporal de las principales variables monetarias muestra que en todos los períodos se produce un crecimiento de los agregados, existiendo una reducción en período de 2007-13 en ambos casos, aunque en el caso español la M1 es sensiblemente menor que la zona euro. Respecto a la velocidad monetaria existe una continua caída siendo mayor la que se produce en el caso europeo.

Una vez analizados la evolución de la masa monetaria, hay que considerar el efecto de las políticas realizadas en el progreso del balance del Banco de España desde el punto de vista de los préstamos netos entre los años 2007 y 2013. Los datos muestran una fuerte incremento del total de préstamos desde los $24,9 \mathrm{~mm}$ de $€$ (2007) hasta los $260,9 \mathrm{~mm}$ de $€$ (2013), aunque el incremento es continuo, el salto se produce en el año 2012 en el que alcanza una tasa de variación interanual del $358 \%$, aunque en el año 2013 cae un $12 \%$.

Tabla 5. Dinámica de las variables monetarias.

\begin{tabular}{lcccccc}
\hline \multirow{2}{*}{ Variables } & \multicolumn{3}{c}{ En la Eurozona (17) } & \multicolumn{3}{c}{ En España } \\
\cline { 2 - 7 } & $\mathbf{1 9 9 6 - 2 0 0 7}$ & $\mathbf{2 0 0 7 - 2 0 1 3}$ & $\mathbf{1 9 9 6 - 2 0 1 3}$ & $\mathbf{1 9 9 6 - 2 0 0 7}$ & $\mathbf{2 0 0 7 - 2 0 1 3}$ & $\mathbf{1 9 9 6 - 2 0 1 3}$ \\
\hline Agregado M1 & 0,091 & 0,054 & 0,078 & 0,163 & 0,013 & 0,113 \\
\hline Agregado M3 & 0,063 & 0,044 & 0,055 & 0,077 & 0,043 & 0,064 \\
\hline PIB/M1 & $-0,069$ & $-0,051$ & $-0,064$ & $-0,127$ & $-0,013$ & $-0,089$ \\
\hline PIB/M3 & $-0,041$ & $-0,040$ & $-0,040$ & $-0,040$ & $-0,043$ & $-0,040$ \\
\hline
\end{tabular}

Nota: Tasas de variación interanuales promedio durante el período estudiado.

Fuente: elaboración propia a partir de los datos del BCE

Tabla 6. Balance del Banco de España (Préstamo neto)

\begin{tabular}{ccccccccccc}
\hline \multirow{2}{*}{ Año } & \multicolumn{2}{c}{ Total } & \multicolumn{3}{c}{ Operaciones Monetarias } & \multicolumn{3}{c}{ Facilidades Permanentes } \\
\cline { 2 - 12 } & \multicolumn{2}{c}{} & \multicolumn{2}{c}{ OPF } & \multicolumn{2}{c}{ OFLP } & \multicolumn{2}{c}{ Crédito } & \multicolumn{2}{c}{ Depósito } \\
\cline { 2 - 12 } & Vol. & T.V. & Vol. & T.V. & Vol. & T.V. & Vol. & T.V. & Vol. & T.V. \\
\hline 2007 & 24.964 & & 18.142 & & 7.617 & & 0,22 & & 35,10 \\
\hline 2008 & 49.230 & 0,97 & 20.998 & 0,16 & 35.903 & 3,71 & 62,34 & 282,36 & 6.745 & 191,17 \\
\hline 2009 & 72.953 & 0,48 & 15.614 & $-0,26$ & 65.137 & 0,81 & 8,81 & $-0,86$ & 7.122 & 0,06 \\
\hline 2010 & 89.077 & 0,22 & 12.135 & $-0,22$ & 86.517 & 0,33 & 5,32 & $-0,4$ & 9.208 & 0,29 \\
\hline 2011 & 64.370 & $-0,28$ & 26.558 & 1,19 & 43.960 & $-0,49$ & 56,2 & 9,56 & 6.040 & $-0,34$ \\
\hline 2012 & 294.981 & 3,58 & 35.672 & 0,34 & 294.077 & 5,69 & 0,59 & $-0,99$ & 34.960 & 4,79 \\
\hline 2013 & 260.905 & $-0,12$ & 25.523 & $-0,28$ & 247.808 & $-0,16$ & 0 & -1 & 12.426 & $-0,64$ \\
\hline
\end{tabular}

Nota: El volumen (vol.) es la media anual medida en Millones de Euros. La T.V es la tasa de variación interanual promedio en tanto por uno.

Fuente: Ibídem.

Desde el punto de vista desagregado, se observa que las operaciones principales de financiación (OPF) se mantienen en niveles próximos al año 2007, siendo su mayor incremento en el 2011 del 112\%, pero presenta caídas en tres de los seis años 
Cárdenas, Luís. Análisis de la gestión monetaria del BCE desde un enfoque de endógeno.

estudiados. Por lo tanto, aunque las OPF presentan un crecimiento de media, no es la principal vía de inyección de liquidez. Por el contrario las operaciones de financiación a más largo plazo (OFLP) son las que muestran un mayor crecimiento, alcanzando un incremento interanual del $569 \%$ en el año 2012 , por lo tanto, el instrumento que ha resultado más efectivo en la expansión del balance han sido las OFLP, especialmente en su forma de medida no convencionales que consisten en una sensible ampliación de los plazos de vencimiento.

La explicación de este incremento hay que buscarla en las facilidades permanentes de crédito, que tanto durante la crisis financiera en el 2008 (aunque estas se colocaron en gran medida en las de depósito que también aumentaron un 191,7\%) como en el año 2011 sufrieron un rápido incremento debido a una mayor demanda de fondos, siendo esta una posible explicación de esta expansión. Obsérvese que en el 2012 también aumenta de forma notable el volumen de depósitos en el Banco de España actuando como drenaje de la liquidez, este comportamiento se corrige en el 2013 con una nueva reducción de los tipos oficiales y el establecimiento del tipo de interés del depósito en el cero por ciento.

Una vez analizado el efecto en el balance del Banco de España, es necesario determinar las consecuencias para los mercados interbancarios y financieros las medidas realizadas, para ello se consideran los diferenciales respecto al tipo de interés oficial fijado por el BdE (Tabla 7), utilizando los bonos a 10 años de España y de Alemana, el Euribor a 3 y 12 meses y el tipo del mercado monetario EONIA (Euro OverNight Index Average), se pueden distinguir los principales rasgos durante la crisis financiera. Los resultados muestran claramente la crisis de deuda soberana en el caso español frente al de Alemania, a partir del año 2009 se cambia la tendencia ascendente de ambos países y Alemania empieza a reducir sus diferencias respecto al tipo oficial llegando a situarse en el $0,67 \%$ (superior al oficial) de media en los meses de julio a diciembre de 2012, por el contrario la remuneración de los bonos españoles en el mismo período alcanza el 5,28\%.

Tabla 7. Diferenciales respecto al tipo oficial del BCE.

\begin{tabular}{ccccccc}
\hline Año & BCE & Bonos Esp. & Bonos Ale. & Euribor 3 & Euríbor 12 & EONIA \\
\hline 2007 & 3,854 & $-0,453$ & $-0,374$ & $-0,423$ & $-0,596$ & 3,468 \\
\hline 2008 & 3,854 & $-0,521$ & $-0,144$ & $-0,780$ & $-0,959$ & 3,468 \\
\hline 2009 & 1,229 & $-2,794$ & $-2,040$ & 0,001 & $-0,389$ & 1,158 \\
\hline 2010 & 1,000 & $-3,298$ & $-1,782$ & 0,189 & $-0,350$ & 0,957 \\
\hline 2011 & 1,250 & $-4,207$ & $-1,404$ & $-0,141$ & $-0,756$ & 1,163 \\
\hline $2012(1)$ & 1,000 & $-4,708$ & $-0,712$ & 0,131 & $-0,478$ & 0,965 \\
\hline $2012(2)$ & 0,750 & $-5,287$ & $-0,673$ & 0,473 & 0,006 & 0,740 \\
\hline $2013(3)$ & 0,667 & $-4,148$ & $-0,801$ & 0,458 & 0,128 & 0,659 \\
\hline $2013(4)$ & 0,500 & $-4,196$ & $-1,129$ & 0,279 & $-0,025$ & 0,491 \\
\hline $2013(5)$ & 0,500 & $-4,004$ & $-1,305$ & 0,274 & $-0,042$ & 0,492 \\
\hline
\end{tabular}

Nota: Expresado en tanto por ciento. Los datos de los bonos españoles y alemanes son la remuneración de la deuda pública emitida a 10 años. Los períodos desagregados son las medias de (1) de enero a junio de 2012; (2) julio a diciembre de 2012; (3) de enero a junio de 2013; (4) julio de 2013 y (5) agosto de 2013.

Fuente: Ibídem. 
Cárdenas, Luís. Análisis de la gestión monetaria del BCE desde un enfoque de endógeno.

Respecto a las variables del mercado interbancario, el comportamiento es sensiblemente diferente, mientras que el Euribor a 3 meses cambia su tendencia (mayor que el tipo OPF) pasando a encontrarse por debajo de esa referencia (excepto en el año 2011 que vuelve a ser superior), el Euribor a 12 meses es superior al del BCE en prácticamente todo el período, excepto en el segundo semestre del 2012 y el primero del 2013, pasando a ser de nuevo superior en los meses posteriores, la falta de datos no permite analizar si el año 2013 será un año de financiación interbancaria menor al oficial, pero los meses de julio y agosto no parecen confirmar esta tendencia.

Por otra parte, la evolución del EONIA, no sufre un cambio en su signo, permaneciendo siempre en un tipo inferior al fijado por el BCE, aunque si se observa una mayor cercanía en los años 2012 y 2013, esto es un resultado esperado pues el banco central a continuado aproximando los tipos a cero por ello el margen de fluctuación es menor, hay que considerar que en los últimos meses el EONIA se ha situado en un $0,008 \%$.

En síntesis, los programas de compra de deuda pública no han resuelto el problema de los diferenciales en la remuneración de los bonos soberanos ni los ha aproximado al tipo oficial ni al de Alemania, aunque entre el primer y el segundo semestre del 2012 se produce una caída de casi un 1\%, pudiendo considerarse efecto del SMP. Respecto al mercado interbancario hay una reducción del Euribor a 3 meses pero no se producen cambios en el Euribor a 12 meses, confirmándose el resultado apuntado por Corbatón, Molina y Sardá (2011) de falta de atipicidad en el mercado interbancario. Por último, el EONIA continúa siendo inferior al tipo de OPF pero aproximándose cada vez más a cero, i.e., los tipos han bajado pero no se ha recuperado el volumen de negociación.

\subsection{La evolución de los precios}

La inflación considerada mediante el Índice de Precios al Consumo Armonizado (HCPI) en la Eurozona, en Alemania y en España (Gráfico 5) se caracteriza por la existencia de tres períodos distintos. Primero, hasta el año 2000 existían tasas de inflación relativamente altas (respecto a la actualidad) en España y aproximadamente dos puntos menores en la zona euro y tres en Alemania.

Mientras que a partir de la entrada en funcionamiento del euro, la inflación se estabiliza en aproximadamente el $2 \%$, siendo inferior en Alemania y superior en España. Por último, la deflación que se produce a partir del 2007 en todo el área vuelve a converger a niveles ligeramente superiores al $2 \%$ objetivo, concretamente 2,5\% de media en el 2012 en la Eurozona. Podemos concluir que la inflación se ha mantenido en la Eurozona próxima a su valor objetivo.

El análisis de la inflación en España (Gráfico 6) requiere distinguir entre el índice de precios al consumo armonizado (IPCA) y el índice de precios industriales (IPRI), este último muestra una mayor volatilidad durante el período de recesión, al incrementase rápidamente a partir del 2010 y vuelve a caer en mayor medida en la siguiente recesión. 
Cárdenas, Luís. Análisis de la gestión monetaria del BCE desde un enfoque de endógeno.

Gráfico 5. Inflación en la Eurozona

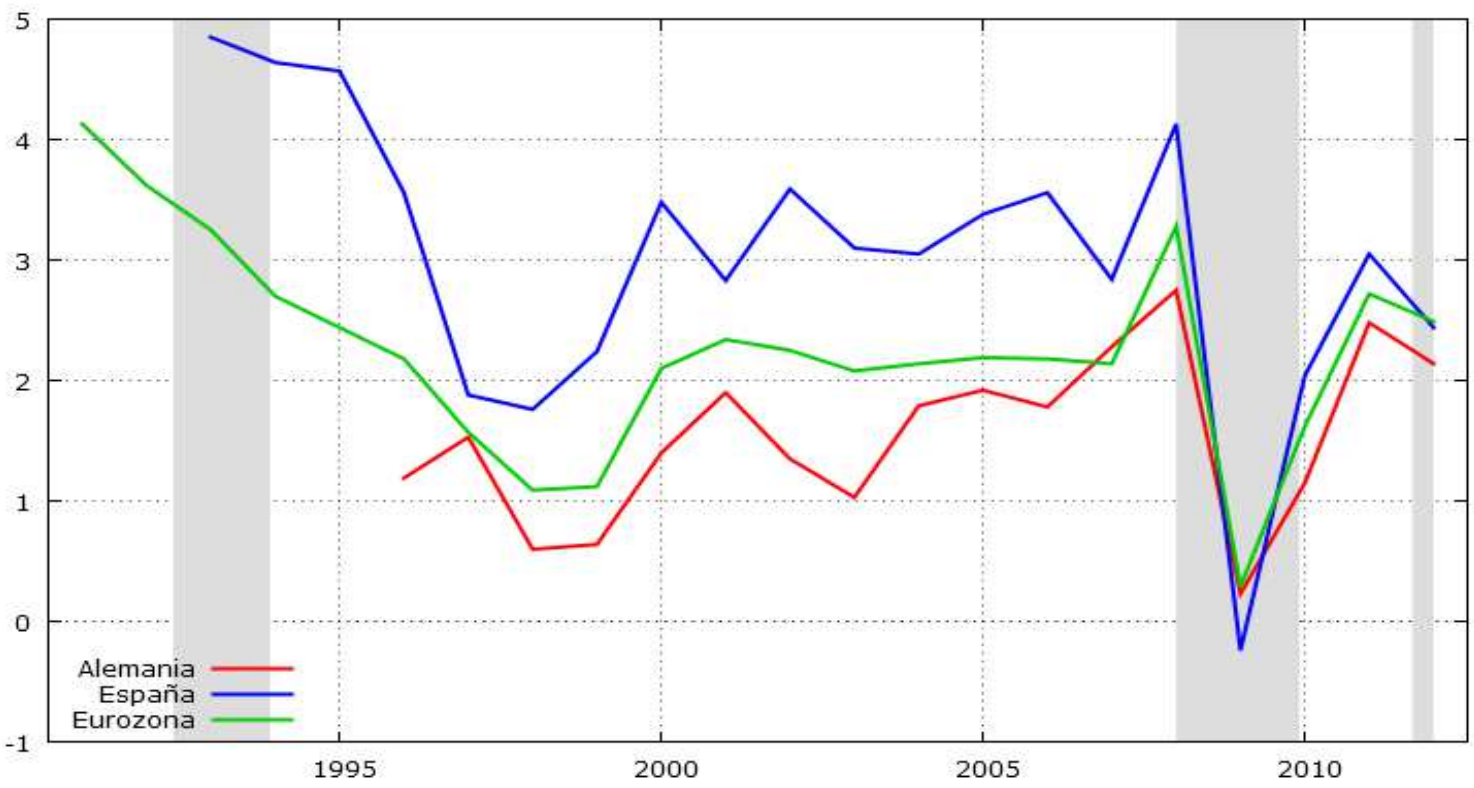

Nota: Se ha utilizado la serie de inflación armonizada. Las zonas sombreadas indican recesión en España.

Fuente: elaboración propia a partir de los datos facilitados por el Banco Central Europeo.

Gráfico 6. Inflación en España.

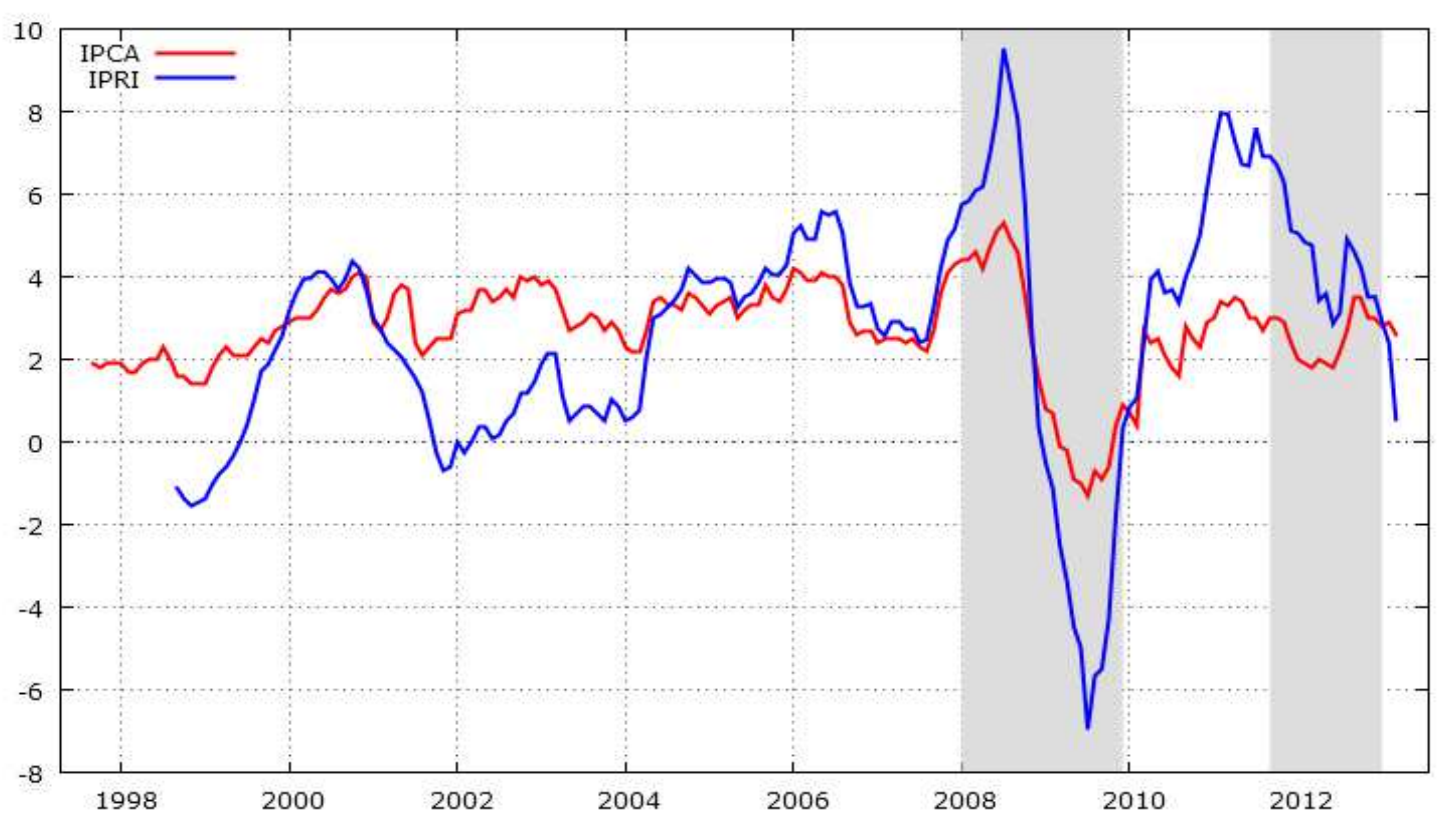

Nota: Se ha utilizado la tasa de variación interanual de la serie de inflación armonizada (IPCA) $y$ el indice de precios industriales (IPRI), ambos en periodicidad mensual. Las zonas sombreadas indican recesión en España.

Fuente: Elaboración propia a partir de los datos facilitados por el ํo de Economía y Competitividad, 2013.

Pero el comportamiento de los precios no ha sido homogéneo durante la crisis financiera y las medidas de actuación del BCE, un estudio más desagregado (Tabla 8) muestra que la mayoría de los precios (tanto de consumo como industriales) cayeron en el año 2009, la excepción son los precios industriales de los bienes de 
Cárdenas, Luís. Análisis de la gestión monetaria del BCE desde un enfoque de endógeno.

equipo que siguen un proceso inflacionario durante todo el período, aunque ralentizado a partir del 2009.

Se observa que la mayor volatilidad de precios se encuentra en el IPC de Alimentos no elaborados que caen un $5,5 \%$ en el 2009 , pero crecen a tasas superiores en los años posteriores. Mientras que los bienes de consumo duradero siguen una tónica deflacionista durante las recesiones, el precio de consumo de la energía se mantiene en niveles positivos para todo el período (excepto el 2009) explicando en gran medida las variaciones del índice general, si se considera que mantiene las tasas de crecimiento más elevadas (alcanzan el $14,7 \%$ en el 2011 ) solo comparables a los precios industriales de la energía (un $14.2 \%$ en el mismo año).

Tabla 8. Evolución de la inflación en España.

\begin{tabular}{cccccccc}
\hline \multirow{2}{*}{ Año } & \multicolumn{3}{c}{ Índice de Precios al Consumo } & \multicolumn{2}{c}{ Índice de Precios Industriales } \\
\cline { 2 - 8 } & General & Alimentos & Duraderos & Energía & General & Equipo & Energía \\
\hline 2007 & 0,027 & 0,031 & 0,003 & 0,017 & 0,035 & 0,033 & 0,016 \\
\hline 2008 & 0,040 & 0,081 & $-0,007$ & 0,112 & 0,063 & 0,025 & 0,132 \\
\hline 2009 & $-0,003$ & $-0,055$ & $-0,025$ & $-0,093$ & $-0,034$ & 0,008 & $-0,069$ \\
\hline 2010 & 0,018 & 0,073 & $-0,008$ & 0,118 & 0,036 & 0,011 & 0,097 \\
\hline 2011 & 0,032 & 0,099 & 0,008 & 0,147 & 0,067 & 0,010 & 0,142 \\
\hline 2012 & 0,024 & 0,062 & $-0,009$ & 0,085 & 0,037 & 0,005 & 0,092 \\
\hline 2013 & 0,022 & 0,025 & $-0,015$ & 0,019 & 0,010 & 0,002 & $-0,013$ \\
\hline
\end{tabular}

Nota: Tasas de variación media anual de los índices de precios en tanto por uno. El año 2013 sólo recoge hasta el mes de mayo por falta de datos.

Fuente: Ibídem.

Para comparar estos dos índices (IPC y IPRI) y la tasa de crecimiento de la oferta monetaria en la Eurozona se muestra en la Tabla 9 la dinámica del ciclo, el resultado más significativo es que la tasa de variación de $\mathrm{M} 1$ es claramente contraria a la inflación, de tal forma que la expansión de esta variable no está teniendo los efectos inflacionistas previstos.

La explicación inmediata a esta circunstancia es que las otras magnitudes monetarias si son positivas, y por tanto, mantienen la habitual correlación respecto al índice de precios; de tal forma que es el mecanismo de transmisión de un agregado a otro el que impide que se produzca la inflación. Se aprecia además que el índice de precios industriales es mucho más volátil que la inflación (4 veces más), siendo este sector más dado a cambios de precios por efectos del ciclo, como incrementos en el precio de las materias primas, especialmente el petróleo, y shocks en la oferta y la demanda, así como de respuesta a la coyuntura.

Asimismo, para el caso de España la volatilidad de la inflación en bienes de consumo es mayor, y la volatilidad de los precios industriales es ligeramente menor, por ello la volatilidad relativa del IPRI español pasa a 2,46 desde el 4,03 europeo, teniendo además una correlación mayor que el índice de consumo e industrial que el europeo. Respecto a los agregados monetarios, de igual forma que en la tabla anterior, es decir, a nivel del euro, la M1 tienen el sentido inverso respecto a los precios de consumo, mientras que la M3 es claramente positiva. Esta característica 
Cárdenas, Luís. Análisis de la gestión monetaria del BCE desde un enfoque de endógeno.

indica que el aumento de la masa monetaria de menor nivel no está teniendo efectos inflacionistas sobre el consumo a nivel cíclico.

Tabla 9. Dinámica Cíclica de la Inflación y la Masa Monetaria, 1997-2012.

\begin{tabular}{cccccccc}
\hline \multirow{2}{*}{ Variable } & \multirow{2}{*}{ Volatilidad } & Volatilidad & \multicolumn{5}{c}{ Correlación Cruzada sobre IPCA(2) } \\
\cline { 5 - 8 } & & Relativa & $X_{t+2}$ & $X_{t+1}$ & $X_{t}$ & $X_{t-1}$ & $X_{t-2}$ \\
\hline IPCA & 0,605 & 1 & 0,7978 & 0,9105 & 1,00 & 0,9105 & 0,8 \\
\hline M1(1) & 0.025 & 0,041 & -0.4421 & -0.5046 & -0.5515 & -0.5910 & -0.6052 \\
\hline M2(1) & 0.0106 & 0,012 & 0.1990 & 0.2470 & 0.2956 & 0.3448 & 0.4026 \\
\hline M3(1) & 0.014 & 0,0175 & 0.3221 & 0.3648 & 0.4182 & 0.4845 & 0.5477 \\
\hline IPRI & 2,439 & 4,031 & 0,7358 & 0,8215 & 0,8712 & 0,8382 & 0,7674 \\
\hline
\end{tabular}

En España

\begin{tabular}{cccccccc}
\hline \multirow{2}{*}{ Variable } & \multirow{2}{*}{ Volatilidad } & Volatilidad & \multicolumn{5}{c}{ Correlación Cruzada sobre IPCA(2) } \\
\cline { 5 - 8 } & & Relativa & $X_{t+2}$ & $X_{t+1}$ & $X_{t}$ & $X_{t-1}$ & $X_{t-2}$ \\
\hline IPCA & 0,904 & 1 & 0,7754 & 0,9045 & 1,00 & 0,9045 & 0,7754 \\
\hline M1(1) & 0,109 & 0,12 & 0,0550 & 0,0124 & $-0,0339$ & $-0,0839$ & $-0,1142$ \\
\hline M3(1) & 0,023 & 0,025 & 0,1942 & 0,2555 & 0,3111 & 0,3479 & 0,3810 \\
\hline IPRI & 2,228 & 2,464 & 0,7325 & 0,8403 & 0,9060 & 0,8785 & 0,8022 \\
\hline
\end{tabular}

Nota: Propiedades de los segundos momentos de los datos filtrados por HP. (1) En diferencias estacionales logarítmicas (2) Todos los valores tienen valore críticos que son significativamente relevantes.

Fuente: elaboración propia a partir de los datos del Mํo de Economía y Competitividad.

Tabla 10. Dinámica de la Producción Industrial y la Política Monetaria. España.

\begin{tabular}{cccccccc}
\hline \multirow{2}{*}{ Variable } & \multirow{2}{*}{ Volatilidad } & \multirow{2}{*}{$\begin{array}{c}\text { Volatilidad } \\
\text { Relativa }\end{array}$} & \multicolumn{5}{c}{ Correlación Cruzada sobre ISI(1) } \\
\cline { 5 - 8 } & & $X_{t+2}$ & $X_{t+1}$ & $X_{t}$ & $X_{t-1}$ & $X_{t-2}$ \\
\hline ISI & 0,041 & 1,00 & 0,855 & 0,938 & 1,000 & 0,938 & 0,855 \\
\hline M1 & 0,040 & 0,975 & $-0,180$ & $-0,025$ & 0,133 & 0,260 & 0,376 \\
\hline M3 & 0,071 & 1,732 & $-0,100$ & $-0,154$ & $-0,211$ & $-0,300$ & $-0,382$ \\
\hline
\end{tabular}

Nota: Propiedades de los datos filtrados en diferencias estacionales logarítmicas. La periodicidad es mensual, tramo considerado desde el 09/2007 al 03/2013 (1) Todos los valores, excepto los dos primeros de la correlación con M1, son significativamente relevantes.

Fuente: elaboración propia a partir de los datos del ํㅡo de Economía y Competitividad.

Si consideramos a nivel nominal, es decir, sin filtrar las series, los resultados cambian sensiblemente, en primer lugar, la volatilidad es ahora muy superior la de los agregados monetarios llegando a un 4,45 la volatilidad relativa de la M1, mientras que respecto al índice industrial apenas varía con respecto al anterior, de ello se deduce que la dinámica entre ambas se explica prácticamente en su totalidad por el componente cíclico.

Otro resultado a considerar es el cambio en la correlación de la M1 respecto al índice de precios de consumo pasando a ser positivo, contrastándolo con el 
Cárdenas, Luís. Análisis de la gestión monetaria del BCE desde un enfoque de endógeno.

resultado anterior se observa que a nivel la respuesta del menor agregado está siendo contraria a los precios, pero a nivel nominal se mantiene la relación positiva habitual aunque la correlación cruzada sea débil en ambos casos.

Este resultado no es diferente al obtenido por Dolado, et al. (1993), que realizaron las primeras estimaciones al respecto, obteniendo un -0,15 y 0,07 de correlación, para la M4 y M1 respectivamente, en términos de fluctuación cíclica, 0,32 y 0,0 en términos de tasa de variación. Por lo tanto, se deduce que las estimaciones realizadas en este trabajo son coherentes con los resultados anteriormente mencionados.

Respecto a la relación entre la producción industrial y los agregados monetarios en el caso español para el período 2007-2013, ha sido claramente contracíclica respecto a la producción, la cual esta medida a través de la variable del Indicador Sintético de Actividad en la Industria (ISI) ajustado. De tal forma que mientras la tasa de variación de la masa monetaria (en especial la M3) aumenta la actividad industrial cae, siendo esta además mucho más volátil que la tasa de crecimiento industrial, las diferencias son menores respecto a la M1, esto se debe probablemente a la política más expansiva del BCE.

En resumen, no se ha producido un efecto expansivo de la actividad ni una reducción del desempleo, como señala el propio BCE, que en su informe sobre el año 2012 afirma:

La frágil confianza económica, el aumento de los precios de las materias primas y el proceso de ajuste de los balances en curso en los sectores financiero y no financiero, sumados a un desempleo elevado, a una orientación fiscal más restrictiva y a una demanda exterior retraída, frenaron la actividad económica en la zona del euro en 2012. Como resultado, el PIB registró un descenso del $0,5 \%$ en el conjunto del año, tras dos años de crecimiento moderado. Debido a la evolución particularmente negativa de la demanda interna, el PIB real de la zona del euro se contrajo a partir del segundo trimestre de 2012, tras el estancamiento del primer trimestre.

\section{EL ENFOQUE ENDÓGENO}

Como se vio en el primer apartado los principios básicos sobre los que se fundamenta la actuación de los bancos centrales se pueden considerar dentro del Nuevo Consenso Macroeconómico (NCM), desde un enfoque endógeno la actuación del BCE no ha sido efectiva 0 no lo ha sido en la medida necesaria por varias razones.

En primer lugar, las actuaciones se empezaron a introducir en un contexto de recesión generalizada, que entre otras cosas llevaron a que por primera vez en la historia contemporánea de España se produjera un período deflacionario. Además, el BCE fue el único banco central importante que intento volver a subir los tipos oficiales, en un intento de ganar aún más credibilidad en su lucha por la estabilidad de precios. Las denominadas medidas no convencionales tienen como principal objetivo la reestructuración del canal de transmisión crediticio.

La centralidad del control de la volatilidad de los precios a corto plazo y la estabilidad de la inflación en el $2 \%$ a largo plazo tienen efectos perjudiciales para la actividad económica; esta política se fundamenta bajo el supuesto de neutralidad monetaria y se fija como único objeto de largo plazo la eliminación o limitación de distorsiones, 
Cárdenas, Luís. Análisis de la gestión monetaria del BCE desde un enfoque de endógeno.

en un intento de permitir la correcta formulación de expectativas por parte de los agentes. Por el contrario, como sostienen Fontana y Palacio-Vera (2007), diversas investigaciones sugieren que la política monetaria tiene efectos incluso a largo plazo, por lo tanto se debe considerar entre sus objetivos el crecimiento de la producción y el empleo en cualquier lapso temporal (a esta política del banco central los autores la denominan "flexible"opportunistic approach)

En tercer lugar, la proximidad de los tipos del BCE a cero (zero lower bound, ZLB) crea un nuevo escenario fácilmente identificable con la tradicional trampa de la líquidez. Frente a la consideración NCM de causación por factores exógenos, desde la perspectiva endógena (Palacio-Vera, 2010) se defiende que bajo condiciones de incertidumbre esta situación puede ser de larga duración si existe un alto grado de preferencia por la liquidez. De lo que se deriva que esta situación puede prolongarse si no se realizan medidas de política expansivas.

La evolución de la inflación no se puede considerar exclusivamente por razones de masa monetaria y tratar de limitarla mediante técnicas de IT, de tal forma que los aumentos en los tipos de interés tienen efectos perjudiciales en la práctica (Arestis y Sawyer, 2006). La explicación debe buscarse en las relaciones de producción, considerando (Palazuelos 2000) los mercados oligopolísticos, los salarios reales, los costes de financiación, el papel de las importaciones o los precios de la energía y el petróleo.

Finalmente, las medidas de política económica que serían necesarias para el crecimiento y el aumento del empleo deben consistir en una serie de reformas socioinstitucionales, que se pueden resumir en:

1. El abandono de las restricciones impuestas por el Pacto de Estabilidad y Crecimiento (la consolidación fiscal) que permita el desarrollo de una política fiscal efectiva actuando tanto en el lado de la oferta como, sobre todo, en el lado de la demanda. Esta cuestión requieren de una reformulación de los principios de gestión estratégica el BCE en el sentido de una orientación monetaria drásticamente diferente a la realizada, incluyendo objetivos de producción y empleo incluso en el largo plazo. En las actuales circunstancias unas expectativas de inflación más elevadas permitirían una reducción indirecta del endeudamiento público y privado que reduciría el proceso de apalancamiento.

2. Los fallos de arquitectura institucional señalados (Malo de Molina, 2011) deben ser objeto de una profunda reforma institucional, con el objeto de limitar la dicotomía entre la evolución de los países periféricos y los centrales, apoyando a países que presenten menores tasa de crecimiento y especialmente ante escenarios de recesión. Según Arestis, 2012, el Banco Europeo de Inversiones (European Investement Bank, EIB) debería tener estos objetivos. Siendo necesarias además formas de coordinación de la política monetaria y fiscal entre los distintos países e instituciones, así como un cambio en las políticas salariales y de precios que tiendan a homogeneizar estos aspectos entre los países de la Eurozona, tendiendo a una mayor consonancia en las fases del ciclo económico.

3. La necesidad de la monetización de deuda soberana, entendiendo por monetización el proceso mediante el cual se transforma un activo financiero, bonos soberanos generalmente, en un incremento de la masa monetaria, siendo dicha operación realizada por el Banco Central, en este sentido ha aportado recientemente evidencia Palacio-Vera (2012). Sus conclusiones son: si no existen 
Cárdenas, Luís. Análisis de la gestión monetaria del BCE desde un enfoque de endógeno.

restricciones a la concesión de préstamos, la monetización tiene un efecto expansivo mediante la política fiscal, además la reducción de los diferenciales con los tipos de interés de referencia también tienen consecuencias positivas. De ello se deriva que la restricción que impone mantener un alto volumen de deuda si este ha tenido que ser financiado a altos tipos de interés tiene un efecto contractivo en la economía, también se deduce que el alto riesgo e incertidumbre en los mercados financieros de deuda pública que ha exigido la intervención del BCE en los mercados secundarios, si se hubiera realizado mediante monetización directa habría tenido, consecuentemente, un efecto estimulante en la economía.

\section{CONCLUSIONES}

En este trabajo se ha resumido la política monetaria del BCE durante la crisis financiera, dicha política ha consistido en una reducción de los tipos de interés oficiales y en las denominadas medidas no convencionales, que consisten en ampliaciones del balance o modificaciones en las adquisiciones de activos. A diferencia de la literatura reciente basada en el NCM, que considera como beneficiosas estas actuaciones, no se observa que estas medidas hayan surtido efecto, incluso las políticas monetarias no convencionales han consistido en una sustitución en los balances pero no en un aumento consistente ni de la producción ni de la masa monetaria, de hecho la correlación entre la producción industrial y la masa monetaria es negativa desde el 2007.

La mayoría de autores señala al sector financiero y al bloqueo del mecanismo de transmisión crediticio como explicación de esta situación, para solucionar esta situación los esfuerzos del BCE se han centrado en paliar la crisis de deuda soberana, en garantizar la liquidez bancaria y en la reactivación del MTM, es en este punto donde se valora positivamente las medidas no convencionales, pero este resultado no es satisfactorio si consideramos la situación de los países periféricos y en concreto el caso español.

La medida que muestra mejores resultados ha sido la ampliación de los plazos de las OFLP, que han expandido sensiblemente el balance del BdE en el año 2012, aunque se observa un descenso con posterioridad, pero estos resultados no muestran una mejoría de la actividad productiva, los tipos de interés de la deuda soberana se mantienen elevados, aunque el Euribor a 3 meses se ha reducido, esto no ha supuesto una reactivación del flujo crediticio ni ha afectado al Euríbor a 12 meses.

Como alternativa al NCM se defiende un enfoque de análisis endógeno, que sostiene que el dinero se encuentra indisociablemente unido al proceso de producción, por tanto se defiende una direccionalidad inversa a la defendida por el NCM, no considerando posible el control cuantitativo de la masa monetaria y de los precios. Las conclusiones obtenidas y las recomendaciones de política económica que se derivan desde esta perspectiva son completamente diferentes a las defendidas por el NCM. Una vez modificada la orientación de estrategia monetaria por parte del BCE, se proponen tres medidas de política económica que podrían desarrollarse. 
Cárdenas, Luís. Análisis de la gestión monetaria del BCE desde un enfoque de endógeno.

Primero, una combinación de política fiscal y monetaria expansivas, sin considerar las restricciones presupuestarias de corto plazo impuestas por acuerdos internacionales, como el Pacto de Estabilidad y Crecimiento, que limitan su efectividad. Creando condiciones monetarias inadecuadas como una trama de líquidez a largo plazo, al poder instrumentarse exclusivamente las reducciones de tipos de interés, en un marco de situación de ZLB. En segundo lugar, una reforma institucional que consolide la actuación de las entidades públicas y permita corregir las dicotomías existentes entre los distintos países de la Eurozona, en especial la relación centro-periferia; con el objeto de homogeneizar las fases cíclicas que permitirían desarrollar con mayor facilidad las políticas públicas.

Por último, ante la gravedad de la crisis de deuda soberana y sus implicaciones para el crecimiento económico y el desempleo, se defiende la posibilidad de monetización de deuda pública, siendo además beneficioso unas expectativas de inflación más elevadas para el lento proceso de desapalancamiento que conjuntamente con los hechos anteriormente enumerados suponen un freno de difícil superación por la Eurozona, y en concreto, en el caso español. 
Cárdenas, Luís. Análisis de la gestión monetaria del BCE desde un enfoque de endógeno.

\section{BIBLIOGRAFÍA}

Alvarez Gil, M. J. \& González-Páramo Martínez-Murillo, J. M. (2012): "La gestión de la crisis financiera global: el papel del Banco Central Europeo", RAE: Revista Asturiana de Economía, 87-102.

Arestis, P. (2011): "Keynesian economics and the New Consensus in macroeconomics", A Modern Guide to Keynesian Macroeconomics and Economic Policies, 88.

Arestis, P. \& Sawyer, M. (2002): "Can monetary policy affect the real economy?", Working Papers Levy Institute

Arestis, P. \& Sawyer, M. (2006): "The nature and role of monetary policy when money is endogenous", Cambridge Journal of Economics, 30, 847-860.

Arestis, P. \& Sawyer, M. (2008): "A critical reconsideration of the foundations of monetary policy in the new consensus macroeconomics framework", Cambridge Journal of Economics, 32, 761-779.

Ayuso, J. \& Malo De Molina, J. L. (2011): El papel de los bancos centrales durante la crisis financiera: lecciones para el futuro. Mecanismos de prevención y gestión de futuras crisis bancarias, Fundación de Estudios Financieros.

Bowdler, C. \& Radia, A. (2012): "Unconventional monetary policy: the assessment", Oxford Review of Economic Policy, 28, 603-621.

Cecioni, M. \& Neri, S. (2011): The monetary transmission mechanism in the euro area: has it changed and why? Temi di Discussione. Working Papers.

Corbatón, J. A., Molina Cobo, C. \& Sardá García, S. (2011): "Análisis de la efectividad de las actuaciones del BCE en el mercado intercambiario durante el período 2007-2010", Boletín económico de ICE, 15-26.

De La Pola Zurita, J. C. (2012): "El pretexto de la inflación. La política alemana de ajustes que privilegia al acreedor", Papeles de Europa, 87-108.

Dolado, J. J., Sebastián, M. \& Vallés, J. (1993): "Cyclical patterns of the Spanish economy", Investigaciones económicas, 17, 445-473.

Dreher, A., Sturm, J.-E. \& Haan, J. D. (2010): "When is a central bank governor replaced? Evidence based on a new data set", Journal of Macroeconomics, 32, 766-781.

Eichengreen, B. \& Temin, P. (2010): "Fetters of gold and paper", Oxford Review of Economic Policy, 26, 370-384.

Esteve, V. \& Prats Albentosa, M. A. (2011): "La utilización de medidas de política monetaria no convencional frente a la crisis financiera internacional", Revista Principios, 19.

Fontana, G. \& Palacio-Vera, A. (2004): "Monetary policy uncovered: theory and practice", International Review of Applied Economics, 18, 25-41.

Fontana, G. \& Palacio-Vera, A. (2007): "Are long-run price stability and short-run output stabilization all that monetary policy can aim for? ", Metroeconomica, 58, 269-298.

Galí, J. (2010): "The monetary pillar and the great financial crisis", Working Papers. Universitat Pompeu Fabra. Departamento de Economía y Empresa.

Giannone, D., Lenza, M., Pill, H., et al. (2010): "Non-standard Monetary Policy Measures and Monetary Developments", CEPR Discussion Papers

Giannone, D., Lenza, M., Pill, H., et al. (2012): "The ECB and the Interbank Market", The Economic Journal, 122, F467-F486.

González-Páramo Martínez-Murillo, J. M. (2012): "La gestión del Banco Central Europeo ante la crisis", Revista de economía mundial, 83-102. 
Cárdenas, Luís. Análisis de la gestión monetaria del BCE desde un enfoque de endógeno.

Hernando, I., Llopis, J. \& Vallés, J. (2012): "Los retos para la política económica en un entorno de tipos de interés próximos a cero", Banco de España.

Howells, P. (2006): The endogeneity of money: empirical evidence, In: Arestis, P. \& Sawyer, M. C. (eds.) A Handbook of Alternative Monetary Economics.

Joyce, M., Tong, M. \& Woods, R. (2011): "The United Kingdom's quantitative easing policy: design, operation and impact", Bank of England Quarterly Bulletin, 51, 200-212.

Krugman, P. R. (1998): "It's Baaack: Japan's Slump and the Return of the Liquidity Trap", Brookings Papers on Economic Activity, 29, 137-206.

Lenza, M., Pill, H. \& Reichlin, L. (2010): "Monetary policy in exceptional times", Economic Policy, 25, 295-339.

Lucas, R. E. (1996): "Nobel lecture: Monetary neutrality", The Journal of Political Economy, 104, 661-682.

Malo De Molina, J. L. (2011): "La crisis y las insuficiencias de la arquitectura institucional de la moneda única", Información Comercial Española, ICE: Revista de economía, 21-34.

Malo De Molina, J. L. (2013): "La respuesta del Banco Central Europeo a la crisis", Boletín económico Julio-Agosto, 115-124.

Millaruelo, A. \& Del Río, A. (2013): "Las medidas de política monetaria no convencionales del BCE a lo largo de la crisis ", Boletín económico, 89-99.

Mishkin, F. S. (2011): "Monetary policy strategy: lessons from the crisis", National Bureau of Economic Research

Palacio-Vera, A. (2010): "The "New Consensus" and the Post-Keynesian Approach to the Analysis of Liquidity Traps", Eastern Economic Journal, 36, 198-216.

Palacio-Vera, A. (2012): "Debt monetization, inflation, and the 'neutral'interest rate", International Review of Applied Economics, 26, 267-285.

Palazuelos Manso, Enrique (2000): "La gestión monetaria en la economía de EEUU: 1981-1996", Boletín económico de ICE, Información Comercial Española, 1327.

Palley, T. (2006): A Post-Keynesian framework for monetary policy: Why interest rate operating procedures are not enough, en Gnos, C. y Rochon, L. P. (Eds.), PostKeynesian Principles of Economic Policy, Cheltenham: Edward Elgar.

Pateiro Rodríguez, C., García Iglesias, J. M. \& Núñez Gamallo, R. (2010): "La gestión de la liquidez del Banco Central Europeo durante la crisis financiera: 2008-2009", Economical Analysis Working Papers, 9, 1.

Pateiro Rodríguez, C. \& García Lorenzo, A. (2002): "El papel del dinero en la estrategia de política monetaria del Banco Central Europeo", Cuadernos de Estudios Empresariales, 49.

Rodil Marzábal, Ó. \& Menezes Ferreira, V. (2012): "La crisis financiera global en perspectivas: Génesis y factores determinantes", Revista de economía mundial, 199-226.

Taylor, J. B. (1993): "Discretion versus policy rules in practice", Carnegie-Rochester conference series on public policy. Elsevier, 195-214.

Taylor, J. B. (2008): "The Financial Crisis and the Policy Responses: An Empirical Analysis of What Went Wrong", National Bureau of Economic Research. 